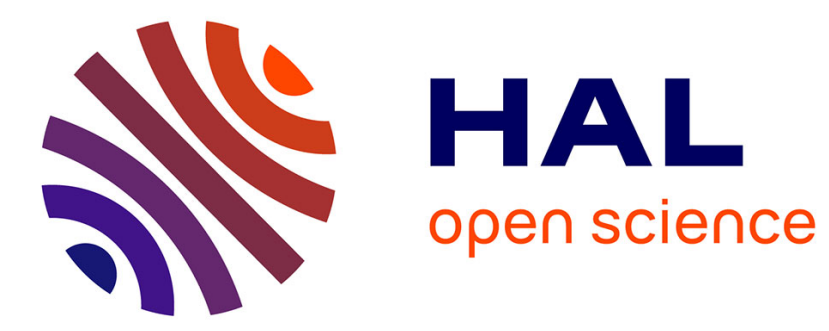

\title{
Local environment dependence of Mn magnetism in bcc iron-manganese alloys: A first-principles study
}

\author{
Anton Schneider, Chu-Chun Fu, Cyrille Barreteau
}

\section{To cite this version:}

Anton Schneider, Chu-Chun Fu, Cyrille Barreteau. Local environment dependence of Mn magnetism in bcc iron-manganese alloys: A first-principles study. Physical Review B: Condensed Matter and Materials Physics (1998-2015), 2018, 98 (9), 10.1103/PhysRevB.98.094426 . hal-01882224

\section{HAL Id: hal-01882224 https://hal.science/hal-01882224}

Submitted on 26 Sep 2018

HAL is a multi-disciplinary open access archive for the deposit and dissemination of scientific research documents, whether they are published or not. The documents may come from teaching and research institutions in France or abroad, or from public or private research centers.
L'archive ouverte pluridisciplinaire HAL, est destinée au dépôt et à la diffusion de documents scientifiques de niveau recherche, publiés ou non, émanant des établissements d'enseignement et de recherche français ou étrangers, des laboratoires publics ou privés. 


\title{
Local environment dependence of Mn magnetism in bec iron-manganese alloys: A first-principles study
}

\author{
Anton Schneider, ${ }^{1}$ Chu-Chun Fu, ${ }^{1}$ and Cyrille Barreteau ${ }^{2}$ \\ ${ }^{1}$ DEN-Service de Recherches de Métallurgie Physique, CEA, Université Paris-Saclay, F-91191 Gif-sur-Yvette, France \\ ${ }^{2}$ DRF-Service de Physique de l'Etat Condensé, CEA-CNRS, Université Paris-Saclay, F-91191 Gif-sur-Yvette, France
}

(Received 25 June 2018; revised manuscript received 7 September 2018; published 26 September 2018)

\begin{abstract}
Magnetic behavior of a $3 d$ solute in a ferromagnetic lattice can be very sensitive to local environment, which is the case of manganese in bcc Fe. Body-centered cubic iron-manganese alloys are studied by means of density functional theory in order to elucidate properties of the lowest-energy magnetic states. Multiple magnetic minima are determined even for the simplest case of an isolated $\mathrm{Mn}$ and a Mn dimer in bcc iron. The magnetoenergetic landscape is analyzed within and beyond the collinear magnetic approximation. A direct correlation is identified between the local electronic charge and the local magnetic moment of a Mn solute, being either isolated or forming a small cluster. In particular, the presence of a vacancy near the Mn atom, inducing a local charge decrease, tends to favor the antiferromagnetic Fe-Mn interaction while the presence of an interstitial impurity with a strong electronic hybridization with $\mathrm{Mn}$ can favor a ferromagnetic Fe-Mn interaction. Energetic and magnetic properties of Fe-Mn alloys are systematically investigated for a large range of Mn concentrations. An unmixing tendency is clearly noted. A detailed magnetic analysis suggests the Mn-Mn magnetic interactions to be generally dominant over the Fe-Mn interactions, both exhibiting an antiferromagnetic tendency. The average magnetic moment of the $\mathrm{Mn}$ atoms in locally random alloys tends to be antiparallel (parallel) to lattice $\mathrm{Fe}$ moments for Mn concentrations smaller (larger) than approximately 6 at. \%. The transition concentration is shown to be lowered if considering Mn clustering which is energetically favorable. The unsolved discrepancies between experimental and theoretical predictions on the critical concentration for the Mn magnetic behavior change in Fe-Mn solid solution are discussed in the light of the obtained results.
\end{abstract}

DOI: 10.1103/PhysRevB.98.094426

\section{INTRODUCTION}

Magnetic interaction between a $3 d$ solute and host atoms in a ferromagnetic lattice is known to depend on the solute $d$ band filling. It tends to be antiferromagnetic (AF) for the early $3 d$ elements and ferromagnetic (FM) for the elements with a large $d$-band filling. In between, magnetic properties of solutes with around half $d$-band filled can be extremely sensitive to local chemical and structural environment. Therefore, they are often at the origin of discrepancies between various data from both theoretical and experimental investigations. This is for instance the case of Mn solutes in bcc iron [1] and $\mathrm{Cr}$ solutes in fcc nickel $[2,3]$. In addition, metal alloys presenting a magnetic competition between solute-solute and solute-host atom interactions often exhibit complex magnetic structures. The interplay between local magnetic order and structural and chemical defects have been widely studied in the past few years for $\mathrm{Fe}-\mathrm{Cr}$ systems [4-11]. In particular, previous studies have shown that magnetism in $\mathrm{Fe}-\mathrm{Cr}$ alloys has a crucial impact on various thermodynamic and kinetic properties $[12,13]$. Such behaviors remain poorly understood for Fe-Mn systems, although it is known that pure bulk Mn shows a complex magnetostructural phase diagram $[14,15]$, and there is a strong magnetoelastic coupling in Fe-Mn alloys [16].

From an application point of view, manganese is a common alloying element in austenitic steels, because of its ability to trap sulfur and its desoxidization properties. Depending on its concentration, Mn may provide improved mechanical properties, act against corrosion, and also increase the hot workability of steels by preventing the formation of sulfides. Also, ferritic/martensitic steels containing manganese are especially interesting as structural materials in nuclear applications due to their reduced activation properties. For instance, they are used nowadays in pressurized water reactors vessels (16MND5) and considered as promising candidates for future nuclear fission and fusion technologies (EUROFER97). More recently, manganese is also involved in the emerging field of high entropy alloys (for instance $\mathrm{Cr}-\mathrm{Mn}-\mathrm{Fe}-\mathrm{Co}-\mathrm{Ni}$ [17]).

This work is focused on the body-centered cubic (bcc) phase of Fe-Mn model alloys. According to the existing FeMn phase diagram [18], the bcc structure is the stable phase at low $\mathrm{Mn}$ concentration (for instance up to 5 at. $\%$ at room temperature). It was also shown that this bcc phase can be extended up to 11 at. \% of Mn at room temperature with cold rolling [19]. On the theoretical side, density functional theory (DFT) results suggest that the bcc phase is more stable than face-centered cubic (fcc) and hexagonal close-packed (hcp) phases at $0 \mathrm{~K}$ up to approximately 13 at. \% Mn [20].

The magnetic behavior of $\mathrm{Mn}$ solutes in dilute $\mathrm{Fe}-\mathrm{Mn}$ systems, which is a first step towards the understanding of properties in Fe-Mn alloys with various concentrations, is still unclear despite previous efforts [21,22]. Existing theoretical studies do not all agree on even the magnetic ground state of a single substitutional $\mathrm{Mn}$ atom in bcc Fe. The reported $\mathrm{Mn}$ magnetic moment ranges from -3.0 to $1.0 \mu_{\mathrm{B}}$. Positive and 
negative moments respectively denote an AF and a FM state with respect to the lattice Fe moments. The value seems to be very sensitive to the various DFT approximations such as the parametrization of the GGA exchange-correlation functional and the use of different types of pseudopotentials, as pointed out by Olsson et al. [23]. The large discrepancy may also arise from the presence of two collinear energy minima for a Mn solute in bcc Fe, showing a moment either parallel or antiparallel to the lattice Fe moments, as mentioned in an early theoretical work [1]. These two minima must be taken into account for an accurate description of $\mathrm{Mn}$ magnetism in bcc Fe-Mn alloys. To the best of our knowledge, it is not the case in most of previously published studies, which may be a possible cause for many discrepancies described in the following paragraphs.

Several DFT studies were focused on Mn interaction with another Mn solute, and with point defects and impurities. Systematic studies of $3 d$ solutes in $\alpha$-Fe including Mn show some controversial results. DFT based calculations performed with projector augmented wave (PAW) potentials reveal an attractive behavior between two nearest-neighbors Mn atoms [23,24], whereas another study performed using Vandebilt ultrasoft pseudopotentials (USPP) showed the opposite [25]. As for the isolated $\mathrm{Mn}$, these energetic results need to be discussed considering different magnetic coupling possibilities of the two Mn atoms. It was also shown that a vacancy and a Mn solute exhibit an attractive behavior [23,24,26-29]. Concerning a Mn solute interacting with an interstitial impurity, a DFT-USPP study reported an attraction, with a binding energy between $\mathrm{Mn}$ and a first-neighbor $\mathrm{C}(\mathrm{N})$ interstitial of $0.26 \mathrm{eV}(0.19 \mathrm{eV})$ [30]. This attractive behavior for $\mathrm{C}$ is confirmed by another DFT-PAW study which reports a lower binding energy $(0.12 \mathrm{eV})$ between $\mathrm{Mn}$ and a first-neighbor $\mathrm{C}$ interstitial impurity [24]. However, these studies have not explicitly discussed the magnetic state variation of the $\mathrm{Mn}$ atom. Further investigation is required to address this feature and magnetoenergetic interplay.

Concerning Fe-Mn alloys beyond the extremely dilute limit, it has been shown via DFT that the heat of formation of bcc Fe-Mn is always positive, in a study performed over the whole range of concentration [20]. This finding agrees with earlier data from tight binding-linear muffin tin orbitals (TBLMTO) calculations [21]. These results are consistent with available experimental works [31,32] revealing an unmixing tendency. However, a systematic concentration dependent magnetic analysis is still missing.

Experimentally, some magnetic properties of Fe-Mn alloys have been studied a few decades ago using Mössbauer spectroscopy [33-35], nuclear magnetic resonance (NMR) [3638], small angle neutron scattering [39], and magnetization measurements [40]. The motivation of these studies was to understand the linear decrease of the total magnetization of $\mathrm{Fe}-\mathrm{Mn}$ systems with increasing Mn concentration, following closely the Slater Pauling curve, up to 11 at. \% Mn, before a sharp drop [22,41,42]. Considering the Fe moment as a constant, it was deduced that the average Mn moment ranges between 0.8 and $1 \mu_{\mathrm{B}}$, parallel to the $\mathrm{Fe}$ moments. This simple scenario could explain the linear decrease of the total magnetic moment by a magnetic dilution effect. On the other hand, more recent experiments on bcc Fe-Mn by NMR and neutron diffuse scattering reported an average Mn magnetic moment of -0.82 and $-0.23 \mu_{\mathrm{B}}$ for respective Mn concentrations of 0.8 and 1.8 at. $\%$, whereas some other experimental works showed moments between 0.6 and $1.0 \mu_{\mathrm{B}}$ for a Mn concentration of 2-5 at. \% Mn [43-46]. Compiling these data seem to show a transition from an AF to a FM Fe-Mn coupling tendency with increasing Mn concentration. A quantitative conclusion is however difficult to draw because the experimental methods and sample conditions may be different from one study to another. None of these works offer a systematic study over a large concentration range. Overall, it is noticeable that low concentration results tend to show AF Fe-Mn interactions while higher concentration studies show a FM Fe-Mn coupling tendency. The transition between these two regimes appears at approximately 2 at. \% Mn, even though it may depend strongly on the experimental details.

On the theoretical side, previous results on Fe-Mn magnetic interactions in the alloys also showed some discrepancies. Some calculations performed using TB-LMTO showed negative Mn moments going from -2.5 to $-3.0 \mu_{\mathrm{B}}$ over the 5-20 at. \% Mn concentration range [47]. The latter method also predicted a transition between a large negative moment and a smaller positive one (from -1.5 to $1 \mu_{\mathrm{B}}$ ) [21], although the critical concentration of this transition $(6-8$ at. \% Mn) is overestimated compared to experimental data (most authors report positive values for $\mathrm{Mn}$ concentrations larger than 2 at. \% Mn in experimental studies) [43-46]. Calculations were also performed using the coherent potential approximation with the Korringa-Kohn-Rostoker (KKR-CPA) method and showed a transition between a strong AF state and a weaker FM state at 12 at. \% Mn [22]. However, CPA is known not to be suitable for addressing properties depending on chemical short range order (SRO), which is the case of Mn magnetism in bcc Fe [46]. It is clear that theoretical results do not agree with the experimental data on the AF to FM transition of the Fe-Mn interaction [48]. One possible reason may be due to the presence of multiple magnetic minima in the Fe-Mn alloys, which are not all considered in the calculations. Also, all these studies only considered random solutions while it was shown that bcc Fe-Mn alloys show significant unmixing tendencies $[31,32]$. It is then necessary to investigate the effects of $\mathrm{Mn}$ clustering on the magnetic properties.

This work aims at elucidating the dependence of $\mathrm{Mn}$ magnetism in various local environments in bcc Fe-Mn alloys, namely a Mn solute, being isolated or forming a cluster, and in the presence of vacancies or interstitial impurities, and the dependence of Mn magnetism on the alloy concentration. Based on the obtained results, we also attempt to provide explanations for the existing discrepancies between theoretical and experimental studies.

The paper is organized as follows: In Sec. II the computational details of the DFT calculations are described. The results are reported and discussed in Sec. III. The first part is focused on the behavior of isolated Mn solutes in a bcc iron lattice. The second part concerns the magnetic structures of bcc Fe-Mn solid solutions as a function of Mn concentration. The third part deals with Mn clusters in bcc Fe. Finally, the fourth and the fifth parts are devoted to the interaction between $\mathrm{Mn}$ atoms and respectively vacancies and interstitial impurities $(\mathrm{C}, \mathrm{N}, \mathrm{O})$ and their impact on the Mn magnetism. 


\section{COMPUTATIONAL METHOD}

\section{A. Density functional theory calculations}

First-principles calculations were performed using density functional theory (DFT) with the projector augmented wave (PAW) method [49,50] as implemented in the VASP (Vienna ab initio simulation package) code [51-53]. The results presented in this paper were obtained using the generalized gradient approximation (GGA) for the exchangecorrelation functional in the Perdew-Burke-Ernzerhof (PBE) form [54]. All the calculations are spin polarized. $3 d$ and $4 s$ electrons were considered as valence electrons. The planewave basis cutoff was set to $400 \mathrm{eV}$. Electronic projected densities of states (PDOS) and atomic magnetic moments are obtained by a projection scheme based on the PAW method as implemented in the VASP code. If using the projection on spheres around atoms as a criterion, we checked that a $10 \%$ variation of the sphere radius from half the nearest-neighbor $(1 \mathrm{nn})$ interatomic distance induces at most a $1 \%$ change of the local magnetic moments. The projection operators are evaluated in the reciprocal space.

Supercell calculations were performed to simulate $\mathrm{Mn}$ solutes in bcc ferromagnetic $\mathrm{Fe}$ with and without a vacancy or impurities. Clusters containing up to $15 \mathrm{Mn}$ atoms were simulated using cubic supercells of $4 \times 4 \times 4,5 \times 5 \times 5$, and $6 \times 6 \times 6$ times the lattice parameter of the cubic unit cell $\left(a_{0}\right)$, containing respectively 128,250 , and 432 atom sites. The solid solutions were represented by special quasirandom structures (SQS) [55] in cubic supercells of $3 \times 3 \times 3$ and $4 \times 4 \times 4$ times $a_{0}$, containing respectively 54 and 128 sites. All the calculations were performed at constant volume. For the SQS systems, the lattice parameters of such systems were set according to Vegard's law. The residual stresses of every studied system were checked to remain lower than 10 kbar.

The $k$-point grids used in our calculations were adjusted according to the size of the supercell. They were chosen to achieve a $k$ sampling equivalent to a bcc cubic unit cell with a $16 \times 16 \times 16$ shifted grid, following the Monkhorst-Pack scheme [56]. The Methfessel-Paxton broadening scheme with $0.1 \mathrm{eV}$ width was used [57]. The convergence threshold for the electronic self-consistency loop was set to $\Delta E=10^{-6} \mathrm{eV}$ and atomic relaxations at constant volume were performed down to a maximum residual force of $0.02 \mathrm{eV} / \AA$. We have verified that the magnetic structures and cluster formation energies are well converged with respect to the choice of $k$-point grids and the cutoff conditions. The resulting error bars for energy differences and magnetic moments of $\mathrm{Fe}$ and $\mathrm{Mn}$ are, respectively, $0.02 \mathrm{eV}, 0.01 \mu_{\mathrm{B}}$, and $0.1 \mu_{\mathrm{B}}$. These are mainly associated with the convergence of the plane-wave energy cutoff and the $k$-grid density.

Some of the calculations presented in this paper were performed beyond the collinear magnetism approximation with or without constrained local magnetism as implemented in the VASP code. A modification was added to the code allowing us to only constrain the local moment of certain atoms of the system. In addition, constrained local magnetism was also applied in the collinear approximation using our own modification of the code. Each constrained local moment calculation was performed incrementing the $\lambda$ constraint parameter up to
TABLE I. Fe bulk elementary properties for each phase. $\Delta E$ stands for the energy difference between the considered phase and the ground state (GS) (bcc FM Fe), $a_{0}$ is the optimized lattice parameter, and $\mu_{\mathrm{Fe}}$ is the magnetic moment per atom. Values between parentheses were obtained by Herper et al. [58] using the full potential linear augmented plane-wave method within the GGA approximation and by Jiang et al. [59] using the PAW method within the GGA approximation. Please note that some reference values are deduced from figures if the precise numbers are not given in Refs. [58,59].

\begin{tabular}{|c|c|c|c|c|}
\hline Phase & Mag. & $\Delta E(\mathrm{eV})$ & $a_{0}(\AA)$ & $\mu_{\mathrm{Fe}}\left(\mu_{\mathrm{B}}\right)$ \\
\hline bcc & FM & $\begin{array}{c}0(\mathrm{GS}) \\
(0.00)[58] \\
(0.00)[59]\end{array}$ & $\begin{array}{l}2.832 \\
(2.843)[58] \\
(2.83)[59]\end{array}$ & $\begin{array}{l}2.20 \\
(2.17)[58] \\
(2.20)[59]\end{array}$ \\
\hline bcc & AFD & 0.178 & 2.841 & 2.18 \\
\hline bcc & $\mathrm{AF}$ & $\begin{array}{l}0.442 \\
(0.44)[58] \\
(0.44)[59]\end{array}$ & $\begin{array}{l}2.793 \\
(2.803)[58] \\
(2.79)[59]\end{array}$ & $\begin{array}{l}1.29 \\
(1.25)[58] \\
(1.22)[59]\end{array}$ \\
\hline bcc & NM & $\begin{array}{l}0.475 \\
(0.47)[58] \\
(0.46)[59]\end{array}$ & $\begin{array}{l}2.757 \\
(2.771)[58] \\
(2.76)[59]\end{array}$ & $\begin{array}{l}0.00 \\
(0.00)[58] \\
(0.00)[59]\end{array}$ \\
\hline fcc & AFD & $\begin{array}{l}0.104 \\
(0.10)[58] \\
(0.10)[59]\end{array}$ & $\begin{array}{l}3.544 \\
(3.538)[58] \\
(3.52)[59]\end{array}$ & $\begin{array}{l}1.93 \\
(1.80) \text { [58] } \\
(1.79) \text { [59] }\end{array}$ \\
\hline fcc & $\mathrm{AF}$ & $\begin{array}{l}0.124 \\
(0.10) \text { [58] } \\
(0.11)[59]\end{array}$ & $\begin{array}{l}3.486 \\
(3.503)[58] \\
(3.48)[59]\end{array}$ & $\begin{array}{l}1.27 \\
(1.30) \text { [58] } \\
(1.23) \text { [59] }\end{array}$ \\
\hline fcc & FM (hs) & $\begin{array}{l}0.154 \\
(0.15)[58] \\
(0.16)[59]\end{array}$ & $\begin{array}{l}3.625 \\
(3.643)[58] \\
(3.64)[59]\end{array}$ & $\begin{array}{l}2.57 \\
(2.57)[58] \\
(2.62)[59]\end{array}$ \\
\hline fcc & FM (ls) & $\begin{array}{l}0.155 \\
(0.13)[58] \\
(0.14)[59]\end{array}$ & $\begin{array}{l}3.480 \\
(3.495)[58] \\
(3.47)[59]\end{array}$ & $\begin{array}{l}1.03 \\
(1.02)[58] \\
(0.94)[59]\end{array}$ \\
\hline fcc & NM & $\begin{array}{l}0.163 \\
(0.15)[58] \\
(0.14)[59]\end{array}$ & $\begin{array}{l}3.446 \\
(3.464)[58] \\
(3.45)[59]\end{array}$ & $\begin{array}{l}0.00 \\
(0.00)[58] \\
(0.00)[59]\end{array}$ \\
\hline
\end{tabular}

the obtaining of a satisfactory low penalty energy term (less than one hundredth of the considered energy differences).

All the alloy concentrations given in the paper are expressed as atomic percent.

\section{B. Bulk Fe and Mn properties: Validation of the approach}

First, the described computational method was used to calculate the relevant crystallographic and magnetic structures of pure $\mathrm{Fe}$ and $\mathrm{Mn}$ bulks. The results were then compared with existing literature data in order to validate our approach and to give additional insights into the magnetic structures of bulk Mn. All these results are obtained with collinear magnetism calculations.

Pure iron is widely known to have a ferromagnetic bodycentered cubic ground state. It is correctly predicted by our calculations which lead to a magnetic moment of $2.20 \mu_{\mathrm{B}}$ per atom, at the optimal lattice parameter which is found to be $2.832 \AA$. Elementary properties for other states are also in excellent agreement with previous results [58-61]. A detailed comparison is summarized in Table I. 
TABLE II. Mn bulk elementary properties for each phase. $\Delta E$ stands for the energy difference between the considered phase and the ground state (GS) (AFM $\alpha-\mathrm{Mn}$ ), $a_{0}$ is the optimized lattice parameter, and $\mu_{\mathrm{Mn}}$ is the magnetic moment per atom. Values between parentheses were obtained by Hobbs et al. [14] and Hafner et al. [15] using the PAW method within the GGA approximation. Please note that some reference values are deduced from figures if the precise numbers are not given in Refs. [14,15].

\begin{tabular}{|c|c|c|c|c|}
\hline Phase & Mag. & $\Delta E$ & $a_{0}(\AA)$ & $\mu_{\mathrm{Mn}}\left(\mu_{\mathrm{B}}\right)$ \\
\hline \multirow[b]{2}{*}{$\alpha$} & \multirow{2}{*}{$\mathrm{AF}$} & 0 (GS) & 8.599 & $2.9 / 2.2 / 0.5 / 0.1$ \\
\hline & & (0) [14] & (8.668) [14] & $(2.8 / 2.2 / 1.0 / 0.0)[14]$ \\
\hline \multirow{2}{*}{$\alpha$} & \multirow{2}{*}{$\mathrm{NM}$} & 0.024 & 8.538 & 0.00 \\
\hline & & (0.04) [14] & (8.532) [14] & (0.00) [14] \\
\hline \multirow{2}{*}{$\beta$} & \multirow{2}{*}{ FiM } & 0.074 & 6.005 & $0.5 / 0.1$ \\
\hline & & (0.063) [14] & $(6.007)[14]$ & $(0.3 / 0.0)[14]$ \\
\hline \multirow[b]{2}{*}{$\beta$} & \multirow{2}{*}{$\mathrm{NM}$} & 0.075 & 6.003 & 0.00 \\
\hline & & - & $(6.007)[14]$ & (0.00) [14] \\
\hline bcc & AFD & 0.128 & 2.850 & 1.89 \\
\hline \multirow{2}{*}{ bcc } & \multirow{2}{*}{$c(2 \times 2)$} & 0.145 & 2.815 & 1.49 \\
\hline & & $(0.146)$ [15] & $(2.81)[15]$ & (1.35) [15] \\
\hline \multirow{2}{*}{ bcc } & \multirow{2}{*}{$\mathrm{FM}$} & 0.170 & 2.790 & 0.85 \\
\hline & & (0.17) [15] & (2.80) [15] & $(0.9)[15]$ \\
\hline \multirow{2}{*}{ bcc } & \multirow{2}{*}{$\mathrm{NM}$} & 0.188 & 2.777 & 0.00 \\
\hline & & (0.19) [15] & (2.77) [15] & $(0.00)[15]$ \\
\hline bcc & $\mathrm{AF}$ & 0.251 & 2.800 & 2.45 \\
\hline \multirow{2}{*}{ fcc } & \multirow{2}{*}{$\mathrm{AF}$} & 0.068 & 3.572 & 1.84 \\
\hline & & (0.067) [15] & (3.55) [15] & (1.60) [15] \\
\hline \multirow{2}{*}{ fcc } & \multirow{2}{*}{$\mathrm{NM}$} & 0.106 & 3.495 & 0.00 \\
\hline & & (0.11) [15] & (3.51) [15] & $(0.00)[15]$ \\
\hline
\end{tabular}

Pure manganese is known under various allotropic forms with distinct magnetic orderings. The lowest energy and most common structure is called $\alpha$-Mn. Its 58-atom cubic unit cell contains four sublattices, each one showing (with collinear magnetism) an antiferromagnetic ordering, even though the actual lowest energy state was predicted to exhibit a noncollinear behavior, almost degenerate with the collinear $\mathrm{AF}$ order [14]. $\beta$-Mn is the second most stable crystallographic structure, found at higher temperatures, with 20 atoms per cubic unit cell forming two sublattices. The magnetic structure of $\beta$-Mn is ferrimagnetic (FiM): each sublattice has a ferromagnetic order, opposed to each other with distinct magnitudes [15]. $\gamma$-Mn shows a face-centered cubic cell whose magnetic order is antiferromagnetic [15]. And finally, we predict the body-centered cubic $\delta$-Mn to have a doublelayer antiferromagnetic (AFD) ground state, at variance with the prediction from an earlier DFT study [15] of a $c(2 \times 2)$ in-plane antiferromagnetic ordering. From our results, the AFD magnetic state presents a magnetic moment of $1.89 \mu_{\mathrm{B}}$ per atom at its optimal lattice parameter of $2.850 \AA$, and the previously described $c(2 \times 2)$ in-plane antiferromagnetic state is $0.02 \mathrm{eV}$ per atom more energetic at its own optimal lattice parameter $(2.815 \AA)$. The magnetic moment in this case is found to be $1.49 \mu_{\mathrm{B}}$. Since both these magnetic orders tend to induce tetragonal deformation to the lattice, we have checked that the AFD state remains lower in energy than the $c(2 \times 2)$ state with full shape and volume relaxations of the simulation cells $(\Delta E=0.01 \mathrm{eV})$. The calculated lattice parameters, relative energies, and magnetic moments for all the considered phases, together with the comparison with previous data, are shown in Table II. All our results are in excellent agreement with previous DFT data findings [14,15], the only exception being the lowest energy AFD state of the $\delta$-Mn phase for which no data was found in the literature.

\section{RESULTS AND DISCUSSIONS}

\section{A. Mn solutes in bcc Fe}

The magnetic properties of isolated single-atom Mn solutes in bcc iron show an atypical feature, as described in a previous theoretical study using LMTO Green's functions [1]. Anisimov et al. have indeed noticed two energy minima with distinct Fe-Mn magnetic interactions. The present calculations within a collinear approximation with constrained local magnetism (see Sec. II A for details) confirm the presence of these two energy minima, as shown in Fig. 1. For a Mn concentration of 1.9 at. \% (1 Mn atom in a 54-atom-site $\mathrm{Fe}$ system), the lowest energy minimum is found with a Mn moment of $-1.9 \mu_{\mathrm{B}}$ antiferromagnetically (AF) coupled with $\mathrm{Fe}$ moments in the lattice (referred to as AF-Mn in the following). The other rather flat minimum is found for FM coupled Mn with $\mathrm{Fe}$ moments (referred to as FM-Mn in the following), with a smaller magnitude of approximately $0.7 \mu_{\mathrm{B}}$. In order to verify if the used 54-atom cell is large enough for the $\mathrm{Mn}$ atom to be considered isolated, we studied the convergence of the relative energy between these two states and their respective magnetic moment magnitude as functions of the simulation supercell size. The results are given in Table III. We note from this table that the energy differences between the AF-Mn and the FM-Mn are all similar, the discrepancy being at most 0.03 $\mathrm{eV}$, which is close to the estimated DFT error bar $(0.02 \mathrm{eV}$, see

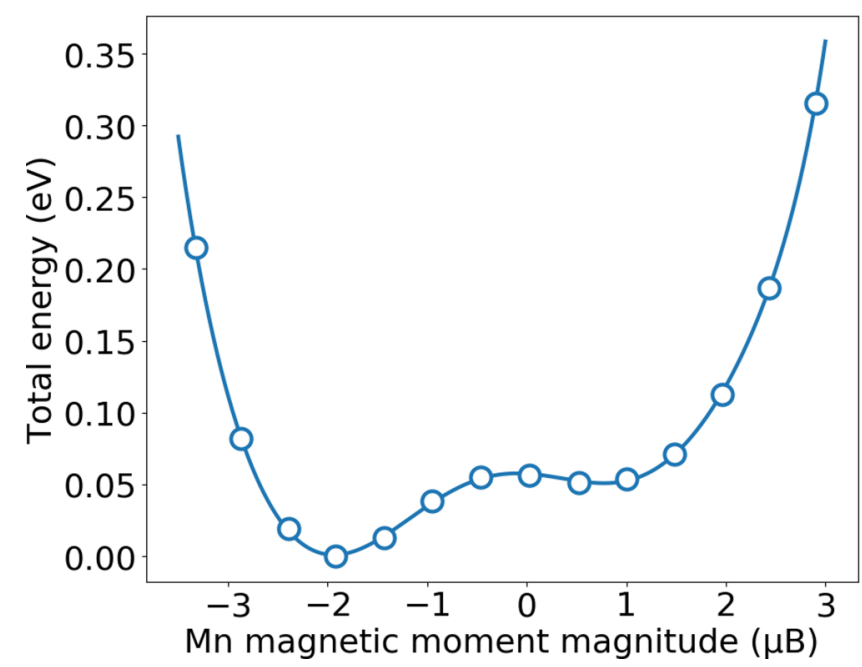

FIG. 1. Total energy as a function of the magnetic moment magnitude of an isolated $\mathrm{Mn}$ atom in bcc Fe. 
TABLE III. Magnetic and energetic properties of an isolated Mn solute in bcc Fe for various supercell sizes resulting in different Mn concentrations. $\Delta E$ is the energy difference between AF-Mn and FM-Mn magnetic states. $\mu_{\mathrm{Mn}-\mathrm{AF}}$ and $\mu_{\mathrm{Mn}-\mathrm{FM}}$ are the magnetic moment magnitudes of the respective AF-Mn and FM-Mn.

\begin{tabular}{|c|c|c|c|c|c|}
\hline System size & Mn (at. \%) & $k$ grid & $\Delta E(\mathrm{meV})$ & $\mu_{\mathrm{Mn}-\mathrm{AF}}$ & $\mu_{\mathrm{Mn}-\mathrm{FM}}$ \\
\hline 54 & 1.9 & $5 \times 5 \times 5$ & 50.7 & $-1.91 \mu_{B}$ & $0.72 \mu_{B}$ \\
\hline 128 & 0.8 & $4 \times 4 \times 4$ & 68.5 & $-1.98 \mu_{B}$ & $0.67 \mu_{B}$ \\
\hline 250 & 0.4 & $3 \times 3 \times 3$ & 52.7 & $-1.94 \mu_{B}$ & $0.85 \mu_{B}$ \\
\hline 432 & 0.2 & $3 \times 3 \times 3$ & 38.4 & $-1.86 \mu_{B}$ & $0.56 \mu_{B}$ \\
\hline
\end{tabular}

Sec. II A). The change of the magnetic moment of the AF-Mn is also close to the DFT error bar $\left(0.1 \mu_{\mathrm{B}}\right.$, see Sec. II A), while the moment of the FM-Mn shows a larger variation. The latter may be due to the shape of the energy minimum, which is rather flat, as shown in Fig. 1. Remaining in the collinear approximation, the estimated energy barrier from the AF to the FM-Mn state is $0.07 \mathrm{eV}$ (Fig. 1).

$\mathrm{Fe}$ and $\mathrm{Mn}$ are close neighbors in the periodic table of elements. As expected, only minor lattice distortion is induced by the substitution of a Mn atom in bcc Fe. In the case of AF$\mathrm{Mn}$, the Fe-Mn $1 \mathrm{nn}$ interatomic distance is very slightly larger than the $1 \mathrm{nn} \mathrm{Fe}-\mathrm{Fe}$ distance in pure $\mathrm{Fe}(\Delta d=0.0025 \AA)$ while in the case of FM-Mn, the $1 \mathrm{nn}$ Fe-Mn distance is shorter compared to the pure Fe bulk $(\Delta d=-0.0144 \AA$ ). Consistently, AF-Mn has a slightly larger Voronoï volume $\left(11.37 \AA^{3}\right)$ than pure $\mathrm{Fe}\left(11.36 \AA^{3}\right)$ which itself has a higher one than FM-Mn $\left(11.20 \AA^{3}\right)$. Also, we notice that the local charge density around the Mn atom is higher in the FM-Mn state than in the AF-Mn state.

Concerning the electronic projected density of states (PDOS), we observe in Fig. 2 that a NM Mn atom in bcc $\mathrm{Fe}$ (from a constrained Mn moment calculation) has a sharp peak on its spin-up PDOS at the Fermi level, indicating (as expected) an instability against the development of magnetism. The spin-down states are mainly concentrated in two peaks located respectively above and below the Fermi level. The AF-Mn state appears by shifting a major part of the spin-down states to lower energies, including some states initially located above the Fermi level, while the main peak of the spin-up band is shifted to above the Fermi level. The PDOS changes are consistent with the high energetic stability of this AF-Mn state. On the other hand, in the FM-Mn case, there is no major difference between the NM and the FM PDOS on the spin-up band, except a slight split of the peak at the Fermi level, decreasing weakly the density of states at the Fermi level. Concerning the spin-down band, a slight shift to higher energies is noticeable, which explains the appearance of a small magnetic moment. The FM-Mn state is only $5 \mathrm{meV}$ below the NM-Mn state.

Noncollinear (NC) magnetism calculations were also performed to explore the possible presence of NC minima. For a $\mathrm{Mn}$ concentration of 1.9 at. \% (1 Mn atom in a 54-atom $\mathrm{Fe}$ system), no other minimum was found besides the two collinear solutions, as shown in Fig. 3. Instead, a significant barrier separates these two minima. One can notice that the noncollinear energy barrier between the two minima is slightly higher than the collinear barrier. Note that the
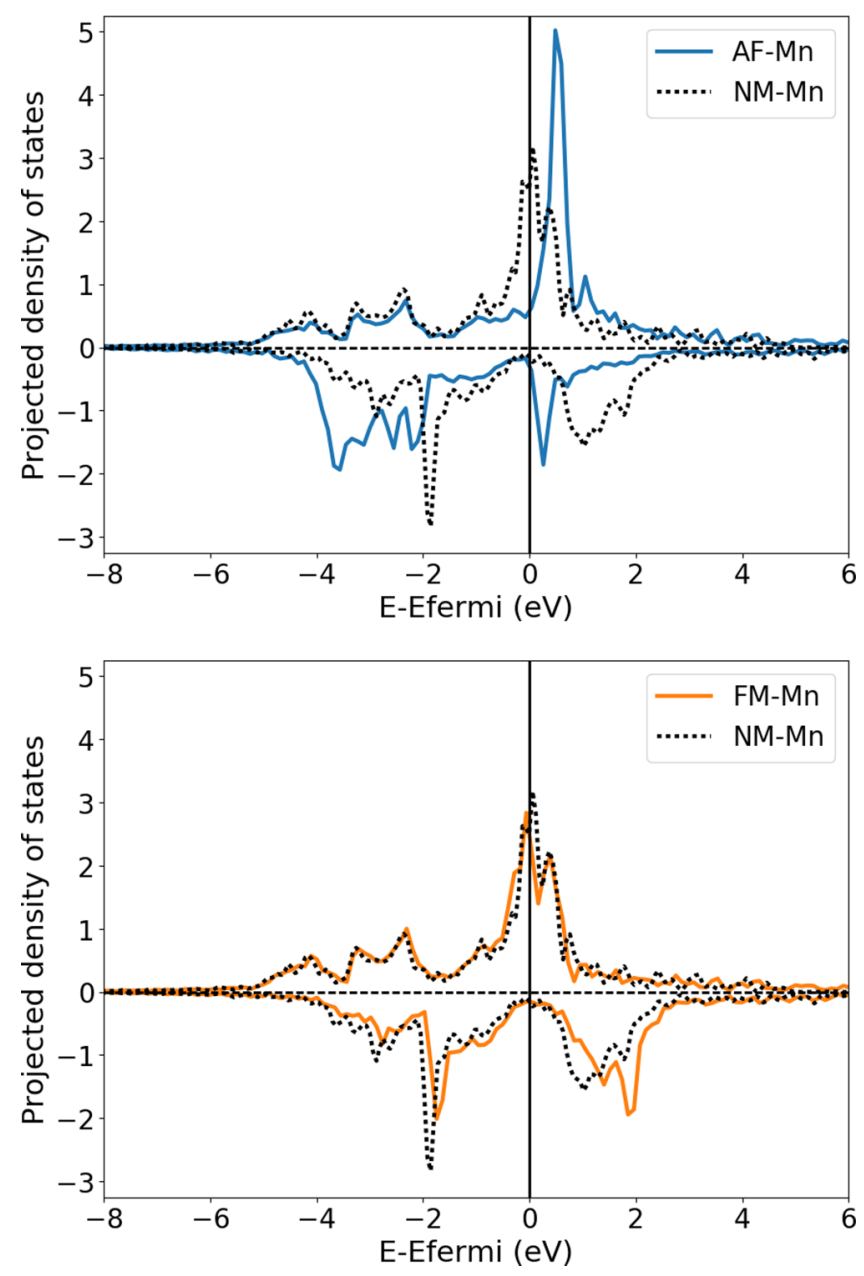

FIG. 2. Projected density of states on an isolated AF-Mn or a FM-Mn, compared to the PDOS on an isolated nonmagnetic Mn atom in bcc Fe.

difference between these barriers is close to the estimated error bar $(0.02 \mathrm{eV}$, see Sec. II A). To calculate this energyangle curve, the direction of the Mn magnetic moment was constrained to certain angles, and we allowed the magnetic moment magnitude to relax. The bottom plot of Fig. 3 shows that, overall, the Mn moment magnitude gradually increases from the FM-Mn state to the lowest energy AF-Mn state, except for the case of $90^{\circ}$ where two energetically degenerate states are found, with a particularly small moment in one of them. These noncollinear data are useful to parametrize Heisenberg-like models for Monte Carlo simulations, in order to describe the magnetic behavior of Fe-Mn alloys at finite temperatures [10].

It is also worth mentioning that the impact of the Mn solute on the local magnetic moment of surrounding $\mathrm{Fe}$ atoms is rather limited. For a Mn concentration of 0.2 at. \% (1 Mn atom in a 432-atom-sites Fe system), the variation of Fe atoms magnetic moment magnitude is at most $5 \%$, similar to what has been shown in $\mathrm{Fe}-\mathrm{Cr}$ alloys [7].

\section{B. Bec Fe-Mn solid solutions}

Most of the present study is devoted to properties of dilute Fe-Mn alloys, because it is known that the bcc Fe-Mn phase 

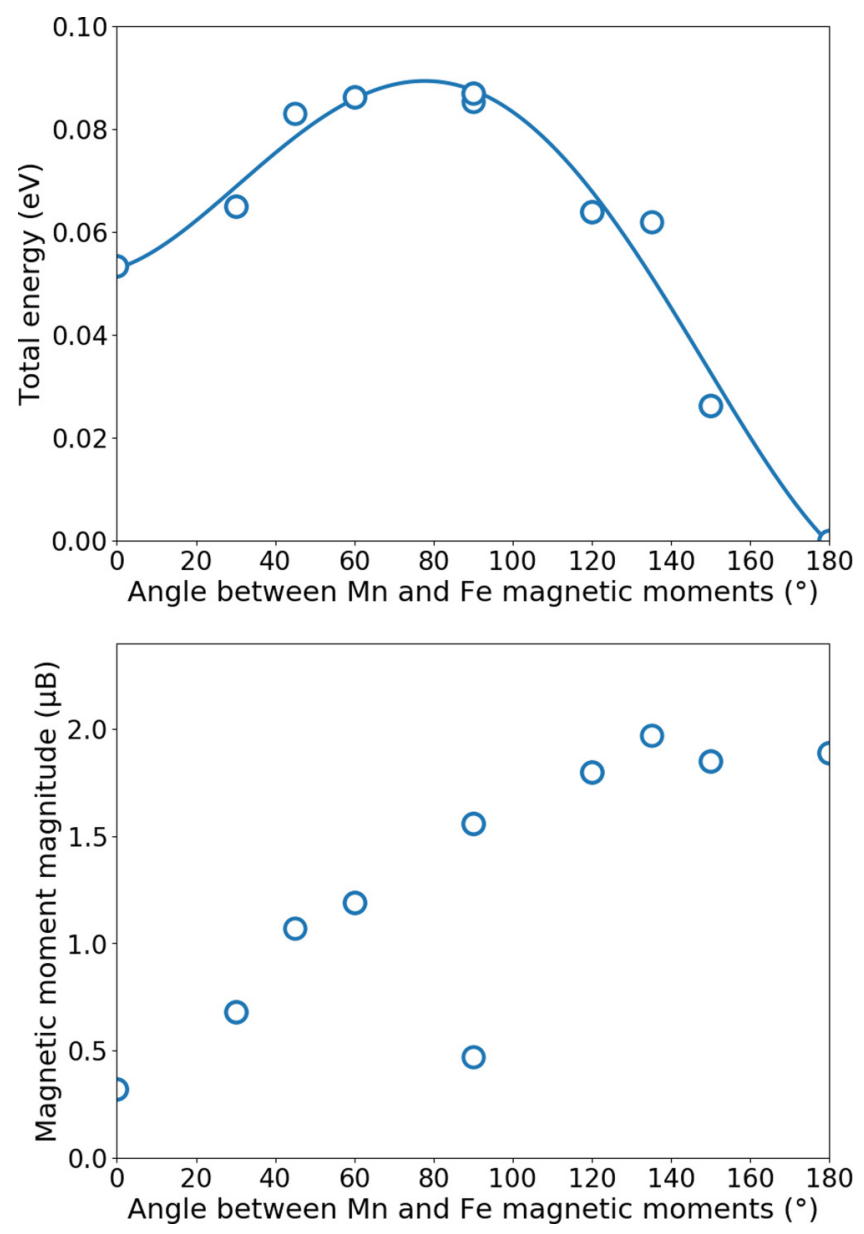

FIG. 3. Total energy (top) and magnetic moment magnitude (bottom) as functions of the angle between moments of an isolated Mn and the lattice $\mathrm{Fe}$ atoms.

is thermodynamically stable only up to a few percent of Mn, before entering in an $\alpha+\gamma$ dual-phase domain [18,20]. However, in order to have a thorough understanding of the dependence of the magnetic behavior on the alloy concentration, it is interesting and relevant to perform a systematic study for all concentrations. Special quasirandom structures (SQS) [55], which have negligible short range order (SRO), are adopted to mimic the random solid solution even with rather small simulation cells. In the SQS systems, Fe and Mn atoms were distributed in 54- and 128-atom cells. Mixing energy of these configurations was calculated using the following expression:

$$
E^{\mathrm{mix}}(\mathrm{Fe}-\mathrm{Mn})=\frac{E^{\mathrm{tot}}(n \mathrm{Fe}+p \mathrm{Mn})-n E(\mathrm{Fe})-p E(\mathrm{Mn})}{n+p},
$$

where $E^{\text {tot }}(n \mathrm{Fe}+p \mathrm{Mn})$ is the total energy of the Fe-Mn solid solution, $E(\mathrm{Fe})$ is the energy per atom of pure bcc $\mathrm{Fe}$ (in its lowest energy magnetic state: FM), and $E(\mathrm{Mn})$ is the energy per atom of pure bcc Mn (in the lowest energy magnetic state: AFD). As can be seen in Fig. 4, the obtained mixing energies are positive for all the concentrations, which reveals an unmixing tendency. Moreover, the shape follows well the regular solution model (shown with the orange curve corresponding to

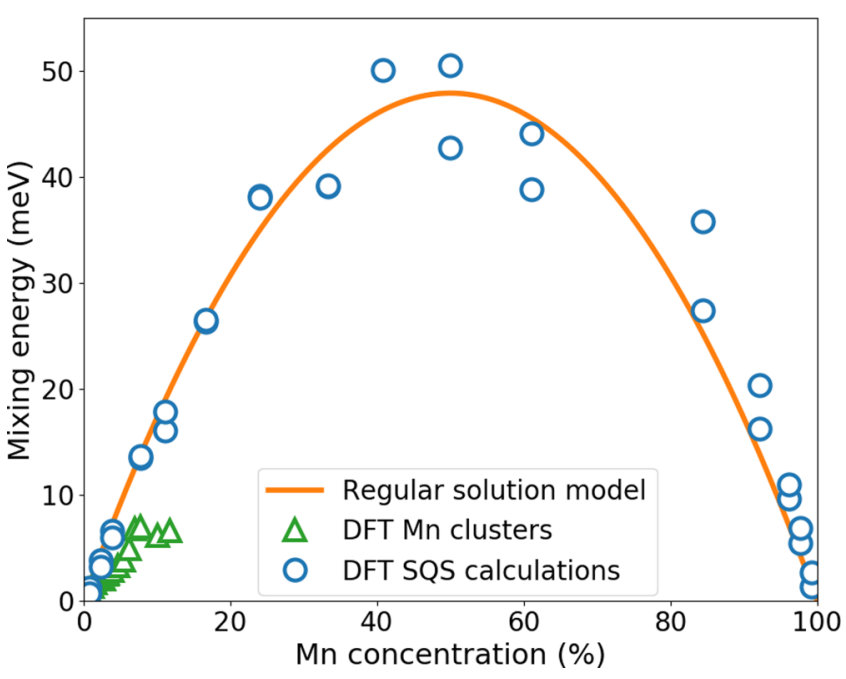

FIG. 4. Mixing energy as a function of Mn concentration (expressed in atomic percent) in bcc Fe-Mn SQS solid solutions and systems composed of Mn clusters in bcc Fe, taking as references the FM bcc Fe and the AFD bcc Mn.

the expression $\left.E^{\mathrm{mix}}=A x_{\mathrm{Fe}} x_{\mathrm{Mn}}\right)$ with $A=192 \mathrm{meV}(18500$ $\mathrm{J} / \mathrm{mol}$ ). It is in good agreement with earlier DFT results showing $A=20000 \mathrm{~J} / \mathrm{mol}[20]$ and an experimental value $(A=19500 \mathrm{~J} / \mathrm{mol})$ [62]. A previous TB-LMTO calculation also obtained a value of the same order of magnitude $(A=$ $23700 \mathrm{~J} / \mathrm{mol}$ ) [21]. In Fig. 4 there are noticeable energy differences between systems at the same concentration with the same atomic configuration but with different magnetic states.

Concerning the magnetic properties, we rather focus on bcc Fe-Mn alloys in the Fe-rich domain, up to 50 at. \% of $\mathrm{Mn} . \mathrm{Fe}$ and Mn local magnetic moments are plotted in Fig. 5 for all the atoms in all the considered SQS configurations up to 50 at. \% $\mathrm{Mn}$. We note a sharp peak for Fe moment around $2.2 \mu_{\mathrm{B}}$ showing a very small perturbation of the bcc-Fe moment due to the presence of $\mathrm{Mn}$ atoms. On the

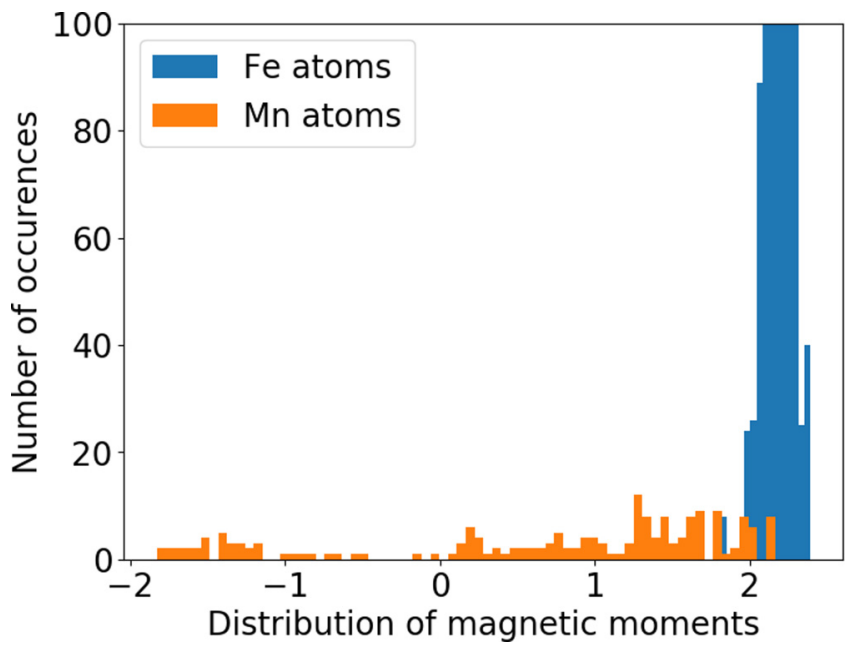

FIG. 5. Distribution of Fe and Mn local magnetic moments in Fe-Mn SQS alloys, up to 50 at. \% Mn. 


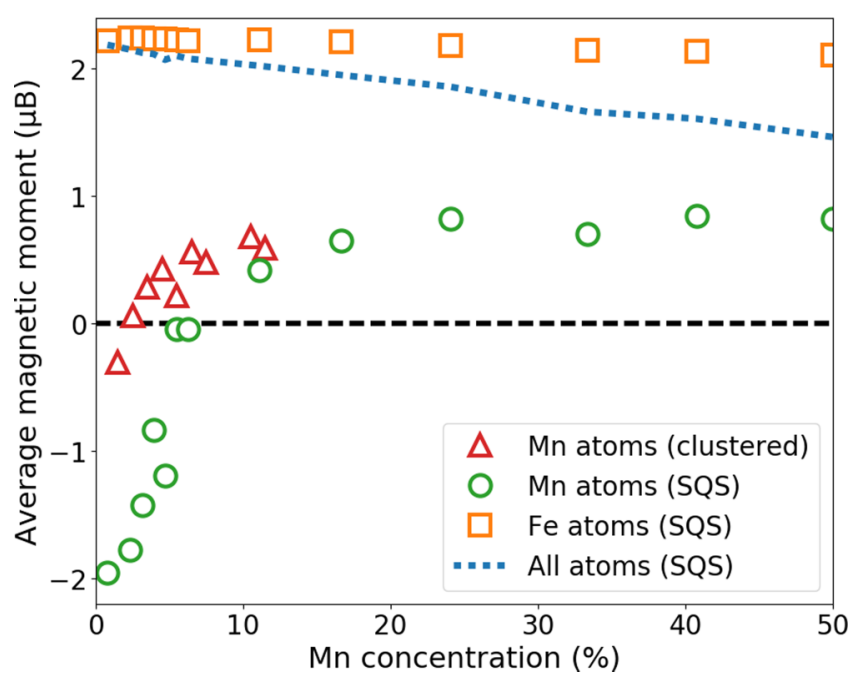

FIG. 6. Average magnetic moment of Fe atoms, of Mn atoms, and of all the atoms in Fe-Mn alloys either with a SQS structure or containing a $\mathrm{Mn}$ cluster, as a function of $\mathrm{Mn}$ concentration (expressed in atomic percent).

other side, Mn moment shows a large dispersion. The average magnetic moment in these SQS solid solutions from Fig. 6 shows a linear decrease with increasing Mn concentration, in agreement with available experimental studies. Indeed, it was shown experimentally that the average magnetic moment of Fe-Mn alloys tend to decrease linearly up to 11 at. \% of $\mathrm{Mn}$, before a sharp drop. It was hypothesized that the linear decrease is due to the magnetic dilution effect (Mn having a constant magnetic moment lower than the Fe moment) and that the sharp drop at higher Mn concentrations is due to the emergence of nonferromagnetic phases [40], but none of these studies led to a final conclusion. From the present results, we suggest that the slope change should correspond rather to a structural phase transition because if the bcc lattice is theoretically kept up to 50 at. \% of Mn as in this study, a linear decrease of the average magnetic moment is observed for all the concentrations, in very good agreement with the SlaterPauling curve. Although we may expect a slight deviation from the linear behavior at low Mn concentration because of the magnetic transition of $\mathrm{Mn}$ atoms (Fig. 6), the effect is not visible.

Having a closer look on the magnetism of Mn atoms, Fig. 6 shows an average Mn moment antiparallel to Fe moments at the lowest concentration, followed by a rapid decrease of the moment magnitude with increasing Mn concentration. This finally leads to a transition from AF to FM Fe-Mn coupling tendency at approximately 6 at. \% Mn (with the SQS systems only), and then the average Mn magnetic moment stabilizes to a value around $0.8 \mu_{\mathrm{B}}$, parallel to Fe moments. As mentioned above, most of $\mathrm{Mn}$ atoms at the dilute limit are isolated and remain in an AF-Mn state. At a high concentration (above 10 at. $\% \mathrm{Mn}$ ) the rather constant value of $0.8 \mu_{\mathrm{B}}$ results from the presence of an approximately constant fraction of $75 \%$ FM-Mn and 25\% AF-Mn atoms in each SQS configuration, as can be seen in Fig. 7. Interestingly, both types of Mn present an average moment magnitude of around $1.6 \mu_{\mathrm{B}}$ (indeed, $1.6 * 0.75-1.6 * 0.25=0.8)$. These features lead to a scenario

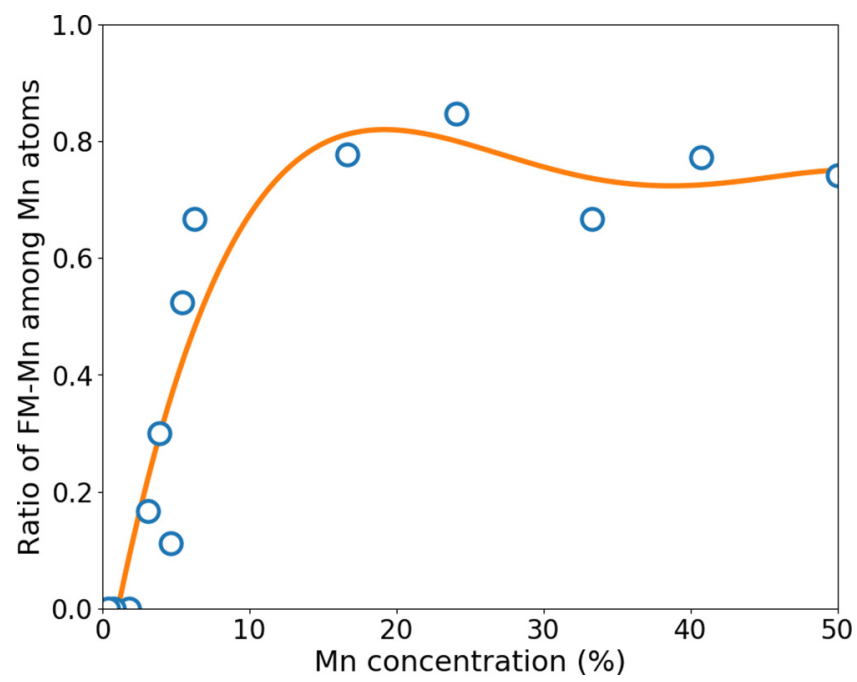

FIG. 7. Ratio of FM-Mn among Mn atoms in Fe-Mn random solutions as a function of $\mathrm{Mn}$ concentration (expressed in atomic percent). The fitted polynomial function (orange curve) aims at illustrating the general trend.

compatible with the simple magnetic dilution model proposed from experimental data, assuming a constant $\mathrm{Mn}$ moment which is parallel and smaller than Fe moments. Of course those experiments have only access to average (instead of individual) magnetic moments [44-46].

If we focus on Mn magnetism in the dilute Fe-Mn alloys, up to a few percent of Mn, some experimental data, summed up in Table IV, tend to show that Mn is coupled FM with Fe between 2 and 9 at. \% of Mn concentrations while another experimental study finds an AF Fe-Mn coupling at Mn concentrations below 2 at. \%. By compiling these results, we note that an AF-Mn to FM-Mn transition is also found in experimental works but at a lower Mn concentration (around 2 at. \% Mn) than our prediction based on SQS systems. Previously, a theoretical study using TB-LMTO method also predicted a transition concentration around 6-8 at. \% $\mathrm{Mn}$, in good agreement with our DFT results [21].

This discrepancy between experimental data and the DFT results may be due to various possible phenomena: For instance, bcc Fe-Mn alloys have an unmixing tendency, the formation of Mn clusters in Fe should be energetically favorable compared to random solid solutions. Indeed, a recent Mössbauer based study [31] reported that bcc Fe-Mn alloys

TABLE IV. Experimental values of Mn average magnetic moment in dilute bcc Fe-Mn for various Mn concentrations.

\begin{tabular}{lcc}
\hline \hline Reference & [Mn] & Avg. $\mu_{\mathrm{Mn}}$ \\
\hline Kajzar et al. [43] & 0.8 at. $\%$ & $-0.82 \mu_{\mathrm{B}}$ \\
Kajzar et al. [43] & 1.9 at. $\%$ & $-0.23 \mu_{\mathrm{B}}$ \\
Child et al. [45] & 2 at. $\%$ & $1.0 \mu_{\mathrm{B}}$ \\
Radhakrishna et al. [46] & 3 at. $\%$ & $0.8 \mu_{\mathrm{B}}$ \\
Radhakrishna et al. [46] & 6 at. $\%$ & $1.0 \mu_{\mathrm{B}}$ \\
Radhakrishna et al. [46] & 9 at. $\%$ & $0.8 \mu_{\mathrm{B}}$ \\
Nakai et al. [44] & 5 at. $\%$ & $0.7 \mu_{\mathrm{B}}$ \\
\hline \hline
\end{tabular}


tend to increase their short range order through clustering. Depending on the thermal treatments and the speed of quenching applied to the experimental samples, the considered Fe-Mn solutions may not be fully disordered. This may be a possible source of discrepancy on the magnetic behavior based on experimental and theoretical studies, if the latter account for SQS solid solutions only. Also, recent DFT works [63] have shown that the presence of carbon impurities in bcc Fe tend to have significant effects on Mn solutes magnetism. The presence of interstitial impurities (often found in iron based alloys) in experimental samples could also affect the magnetic moment of Mn atoms, even though it is not expected to be a major cause because of the generally low impurity content. In addition, the discrepancy may also be related to the presence of structural inhomogeneities in the samples such as grain boundaries and precipitates, but these features are out of the scope of this study. In the following sections we will focus on Mn clustering, vacancy, and impurities effects.

\section{Mn clusters in bcc Fe}

Before addressing Mn clusters, the interaction between two isolated Mn solutes was investigated as a function of the distance between them. It was found that as nearest neighbors $(1 \mathrm{nn})$ or second-nearest neighbors $(2 \mathrm{nn}), \mathrm{Mn}$ atoms tend to be AF with each other. One of them is therefore FM to the lattice $\mathrm{Fe}$ atoms, at variance with the isolated $\mathrm{Mn}$ case. This results from a dominance of Mn-Mn AF tendency over the Fe-Mn antiferromagnetism at such short separation distances. This behavior is opposite to the $\mathrm{Fe}-\mathrm{Cr}$ alloy case, where $\mathrm{Fe}-\mathrm{Cr}$ $\mathrm{AF}$ interactions prevail over the $\mathrm{Cr}-\mathrm{Cr}$ antiferromagnetism [10]. The Mn local moments for the $1 \mathrm{nn}$ separation are $-1.7 \mu_{\mathrm{B}}$ (AF-Mn) and $1.4 \mu_{\mathrm{B}}$ (FM-Mn). For the $2 \mathrm{nn}$ case, the corresponding $\mathrm{Mn}$ moments are -1.8 and $1.1 \mu_{\mathrm{B}}$. Please note that the FM-Mn within the dimer has a significantly increased moment magnitude compared with an isolated FM$\mathrm{Mn}$, especially for the $1 \mathrm{nn}$ case. At a larger Mn-Mn distance, with the two Mn atoms as third-nearest neighbors (3nn) and beyond, both of them prefer to couple AF with Fe moments with magnetic moments converging towards the value of an isolated $\mathrm{Mn}$ in bcc Fe. Figure 8 shows the binding energy between two Mn atoms in bcc Fe as a function of the separation distance for various collinear magnetic configurations. The Mn-Mn binding energy is calculated as follows:

$$
\begin{aligned}
E^{\mathrm{bind}}(\mathrm{Mn}-\mathrm{Mn})= & -E^{\mathrm{tot}}((n-2) \mathrm{Fe}+2 \mathrm{Mn})+2 E^{\mathrm{tot}}((n-1) \\
& \mathrm{Fe}+1 \mathrm{Mn})-E^{\mathrm{tot}}(n \mathrm{Fe}),
\end{aligned}
$$

with $E^{\text {tot }}((n-1) \mathrm{Fe}+1 \mathrm{Mn})$ at the magnetic ground state $(\mathrm{Mn}$ coupled $\mathrm{AF}$ with $\mathrm{Fe}$ ). Using this expression, positive binding energies stand for attractive behaviors, while negative binding energies stand for repulsion. Figure 8 shows that, in the lowest-energy magnetic state, the $1 \mathrm{nn}$ Mn dimer exhibits a very slight attraction $(0.012 \mathrm{eV})$, whereas there is a repulsion from a $2 \mathrm{nn}$ to a $7 \mathrm{nn}$ distance. Note that the magnitude of the attraction is within the error bar of the present DFT calculations $(0.02 \mathrm{eV})$. The binding energy converges to 0 from the $8 \mathrm{nn}, \mathrm{Mn}$ atoms are then considered fully isolated. At a $1 \mathrm{nn}$ distance, the dimer composed of two FM-Mn or two AF-Mn atoms is respectively 0.045 or $0.062 \mathrm{eV}$ higher in energy than the magnetic ground-state dimer (AF-Mn +

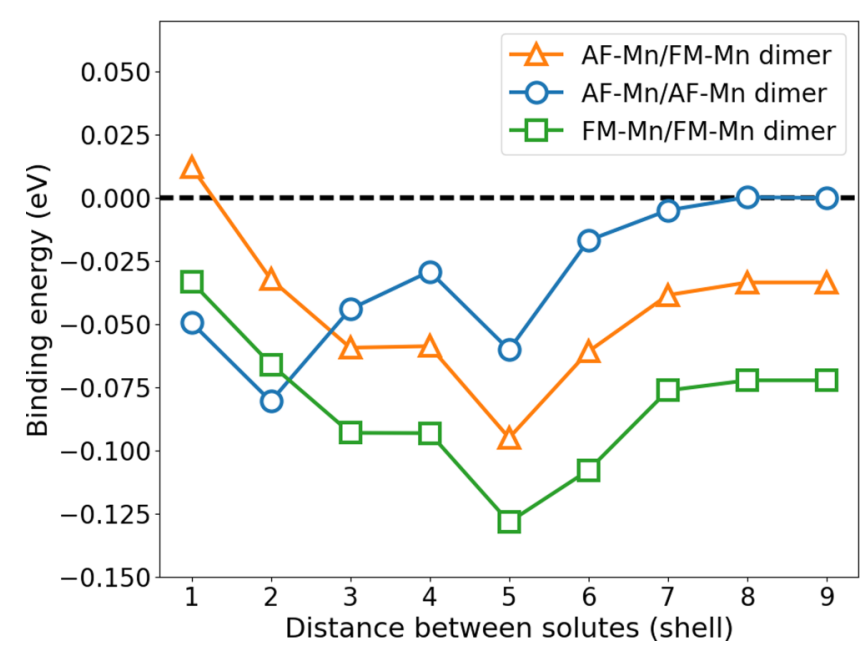

FIG. 8. Binding energy between two Mn atoms in a 432-atom bcc Fe supercell as a function of Mn-Mn separation distance, taking isolated AF-Mn as the reference. Positive binding energies mean attraction.

FM-Mn). The trend remains the same for a $2 \mathrm{nn}$ dimer. It is worth mentioning that the dimer with two AF-Mn comes to have even a slightly higher energy than the dimer with two FM-Mn atoms (0.017 eV higher for the $1 \mathrm{nn}$ case). This scenario may be understood assuming a dominant Mn-Mn AF interaction, over the Fe-Mn antiferromagnetism at these shortest separations: the two AF-Mn dimer (FM coupling between the Mn atoms) exhibiting large Mn moments: $-2.43 \mu_{\mathrm{B}}$ per $\mathrm{Mn}$ for the $1 \mathrm{nn}$ case, is energetically penalizing, while the two FM-Mn dimer develops a magnetically less unfavorable situation by decreasing the moment magnitudes $\left(0.18 \mu_{\mathrm{B}}\right.$ per Mn for the $1 \mathrm{nn}$ case).

Bakaev et al. [24] have noticed that among several substitutional solutes, $\mathrm{Mn}$ is the only one to show attractive solute-solute interactions in bcc $\mathrm{Fe}$ at a $1 \mathrm{nn}$ distance. That study leads to the conclusion that this specificity is mainly due to the flexibility of the Mn magnetic moment, which means an easy variation of the moment magnitude, to adapt different local environments. Our study also reveals such flexibility, as reflected by the existence of distinct moment magnitudes of $\mathrm{Mn}$ in bcc Fe (Fig. 5). Furthermore, as mentioned above, two minima (AF-Mn and FM-Mn) exist for a single Mn solute, which provides an additional possible state for each $\mathrm{Mn}$ in the dimer. To our knowledge, the possibility of a Mn dimer with one AF-Mn and one FM-Mn was not considered in Ref. [24]. Also, the results from Bakaev et al. and the present ones may not be directly comparable, as different parametrization of GGA are used (PW91 in Ref. [24]).

In addition, noncollinear magnetism calculations were performed in the case of $1 \mathrm{nn} \mathrm{Mn}$ atoms to explore the possible presence of noncollinear energy minima, which may provide a compromise between the preference for a single $\mathrm{Mn}$ to be $\mathrm{AF}$ to $\mathrm{Fe}$, and the emergence of a FM-Mn in the dimer, due to Mn-Mn interaction. From our NC calculations, no configuration was found with a lower energy than the collinear magnetic ground state. Figure 9 shows the binding energy for some noncollinear configurations obtained, along with the respective spin configurations. Calculations were performed 


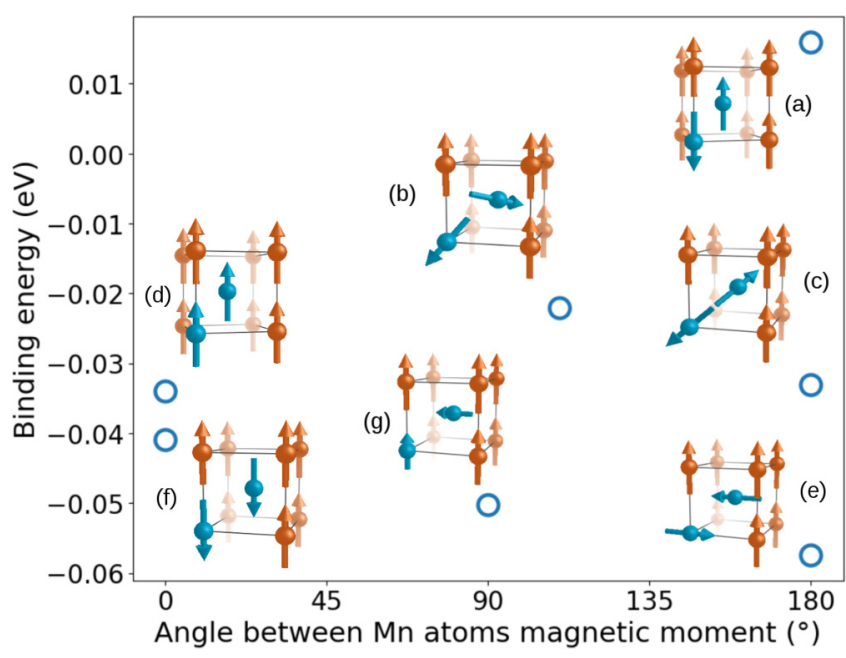

FIG. 9. Binding energy (denoted by blue circles) of a $1 \mathrm{nn} \mathrm{Mn}$ dimer, with the AF-Mn as reference, for various shown collinear and noncollinear magnetic configurations. $\mathrm{Mn}$ and $\mathrm{Fe}$ atoms are, respectively, represented by blue and orange spheres, and orientations of local magnetic moments are indicated with arrows.

with several different initial spin arrangements. All the cases converged to either the collinear ground state (a) or the NC (b) configuration, which seems to be the only stable NC state. At variance, the (c), (g), and (e) configurations were obtained using constrained local magnetism, by imposing the Mn spin directions but relaxing the spin magnitudes. As can be seen in Fig. 9, the NC metastable (b) state is higher in energy than the collinear ground state (lower in binding energy), but less energetic than other collinear states [(d) and (f)]. Also, please note that these energy differences are rather close to the estimated error bar of the calculation method or even fall within it. Moreover, we may expect a non-negligible occupation rate of the metastable states, including the $\mathrm{NC}-(\mathrm{b})$ state at finite temperatures. It is in principle also relevant to perform the study of various-sized Mn clusters beyond the collinear approximation. However, such a systematic NC magnetic study is significantly computationally demanding and out of the scope of this paper. A full noncollinear study will be considered in a future work. The following sections only deal with collinear calculations.

Concerning the study of $n$-Mn clusters in bcc Fe with $n=$ 3 to 15 , we employed 250 -atom supercells and various atomic configurations (more or less compact) and magnetic arrangements were considered for each cluster size. The lowest energy atomic and magnetic configurations of these clusters are shown in Fig. 10. It was found that the average binding energy of these clusters, calculated using the following equation:

$$
E_{\text {avg }}^{\text {bind }}(p \mathrm{Mn})=\frac{-E^{\mathrm{tot}}((n-p) \mathrm{Fe}+p \mathrm{Mn})+p E^{\mathrm{tot}}((n-1) \mathrm{Fe}+1 \mathrm{Mn})-(p-1) E^{\mathrm{tot}}(n \mathrm{Fe})}{p}
$$

is all positive (energetically favorable) and increases with the number of Mn atoms, as shown in Fig. 11. The attraction between $\mathrm{Mn}$ atoms confirms the unmixing tendency showed in the previous section on solid solutions. As can be seen in Fig. 4, for Mn concentrations ranging from 1 to 12 at. \%, mixing energy of Fe-Mn systems containing clusters, in their lowest-energy magnetic state, is indeed lower than the energy of random Fe-Mn alloys with the same Mn content.

Regarding the magnetic ordering of the Mn clusters, it is noticeable from the lowest energy magnetic configurations (Fig. 10) that the Mn-Mn magnetic interaction is dominant compared to Fe-Mn coupling, as already noted for the Mn dimer. Indeed, these magnetic arrangements tend to satisfy in priority the AF Mn-Mn 1nn and 2nn coupling. For example, at least $50 \%$ of $1 \mathrm{nn}$ Mn-Mn pairs in a cluster show an AF coupling. Some $n$-Mn clusters $(n=2,3,4,6,7)$ present $100 \%$ of AF $1 \mathrm{nn}$ Mn-Mn pairs. At variance, Fe-Mn magnetic interaction does not show any clear tendency, the fraction of $1 \mathrm{nn}$ AF Fe-Mn pairs varies from $20 \%$ to $70 \%$. Moreover, switching every $\mathrm{Mn}$ atom magnetic moment (from AF-Mn to FM-Mn and vice versa) changes significantly the number of $\mathrm{AF} \mathrm{Fe}-\mathrm{Mn}$ pairs without changing any Mn-Mn magnetic interaction. It induces rather small energy modifications (less than $10 \mathrm{meV}$ per $\mathrm{Mn}$ ), and the sign of the energy variation shows no dependence on the sign of number variation of AF Fe-Mn pairs. However, switching some Mn magnetic moments (changing the number of AF Mn-Mn pairs) generally leads to unstable states. A particular case is noted in
Fig. 10, the 8-atom cluster, where two atoms have a very small magnetic moment $\left(0.2 \mu_{\mathrm{B}}\right)$ which results certainly from magnetic frustrations between several interactions which cannot all be satisfied within the collinear approximation. Since these clusters are small, they can obviously not develop the AFD structure as in the lowest-energy state of pure bcc Mn, because of finite size effects. However, we notice that in the 15-Mn cluster, the ratios of $\mathrm{AF} 1 \mathrm{nn}$ and $2 \mathrm{nn}$ interactions are, respectively, $75 \%$ and $33 \%$, which are not too far from the values for pure bcc AFD Mn (respectively 50\% and 33\%).

Average magnetic moment magnitudes of the two types of Mn atoms forming the clusters: the AF-Mn and the FM-Mn are shown in Fig. 12. We note a generally larger moment magnitude for the FM-Mn forming a cluster than isolated. The opposite occurs for the AF-Mn atoms in the case of the smallest clusters. The intermediate sized clusters between 7 and 9 atoms show some particular values, mostly lower than the corresponding Mn moments in the considered clusters. This behavior may be due to a transition between the very small clusters (arranged within a cubic unit cell) in which every $\mathrm{Mn}$ atom is an interface atom (mainly surrounded by $\mathrm{Fe}$ atoms) and more extended clusters, in which Mn atoms have a lot of Mn nearest neighbors.

Considering the average Mn magnetic moment of a cluster in its lowest energy magnetic configuration, we observe that the magnetic Fe-Mn coupling tendency shows the same trend as in the random solutions, except that the transition point appears at a lower concentration (around 3 at. \% $\mathrm{Mn}$ in 


\section{- $\mathrm{Mn}$ \\ $\mathrm{Fe}$}
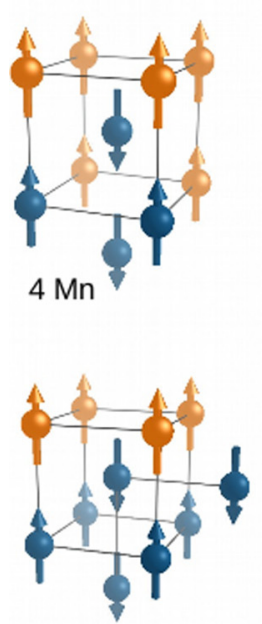

$7 \mathrm{Mn}$
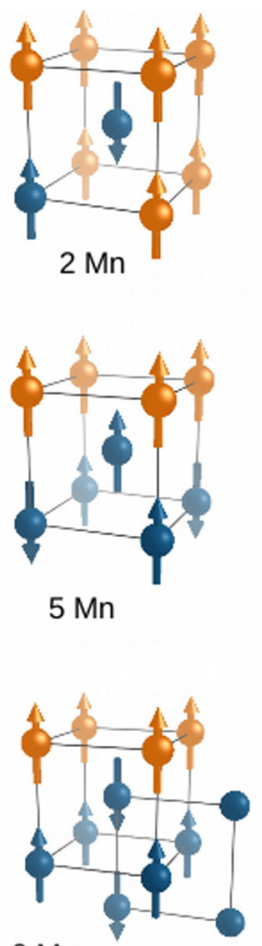

$8 \mathrm{Mn}$
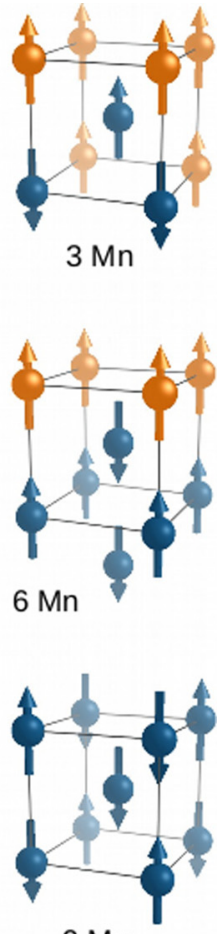

$9 \mathrm{Mn}$

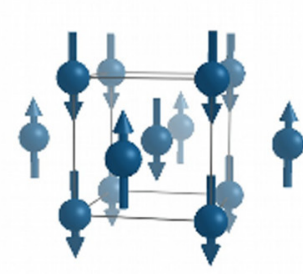

$13 \mathrm{Mn}$

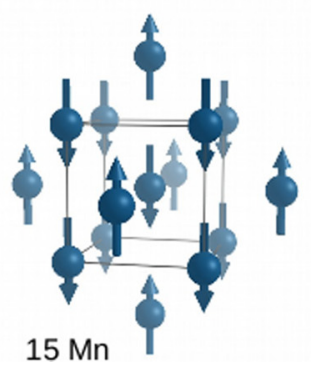

FIG. 10. Schematic representation of the lowest energy atomic and magnetic configuration found for $n$-Mn clusters in a bcc Fe lattice, for $n=2$ to 15 . Magnetic moment orientations are indicated by arrows. In the case of negligible moment magnitudes, atoms are represented without arrows.

Fig. 6). This feature may be one possible explanation of the discrepancy between experimental and theoretical data concerning the transition concentration of the average $\mathrm{Mn}$ moment behavior, from an AF to a FM state with respect to the Fe moments, in Fe-Mn alloys. Figure 6 shows that clusters resulting average $\mathrm{Mn}$ moment is indeed $\mathrm{FM}$ at concentrations for which Fe-Mn random solutions exhibit an AF Fe-Mn average magnetic coupling (for Mn concentrations between 3 and 6 at. \%). Indeed, the existing discrepancy comes from data based on random solid solutions assumed in theoretical calculations [21,22,47], whereas experimental samples may contain $\mathrm{Mn}$ clusters in $\mathrm{Fe}$ which are more stable systems at low temperatures. Of course the detailed composition of the experimental samples is strongly related to the thermal treatments such as the speed of quenching [43-46].

\section{Mn interaction with vacancies in bcc $\mathrm{Fe}$}

Beyond the magnetic and energetic properties of idealized $\mathrm{Fe}-\mathrm{Mn}$ alloys, another focus of this study is the variation of

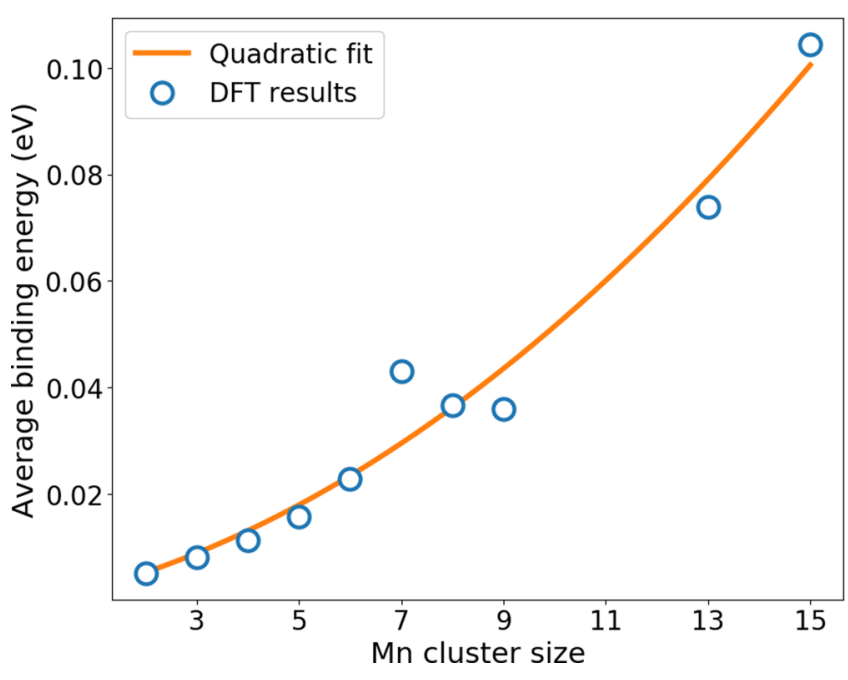

FIG. 11. Average binding energy (per atom) of lowest energy atomic and magnetic configurations of Mn clusters versus the number of $\mathrm{Mn}$ atoms composing the cluster, taking the isolated AF-Mn as reference. Positive values indicate an attraction.

these properties in the presence of point defects, for instance vacancies. First, we have investigated the effects of a vacancy on a single Mn solute in bcc Fe. To do so, a vacancy was inserted in a 128-site bcc Fe lattice containing one $\mathrm{Mn}$ atom (in either AF-Mn or FM-Mn state), at various distances from the $\mathrm{Mn}$ atom, from $1 \mathrm{nn}$ to $6 \mathrm{nn}$. The presence of the vacancy increases the energy gap between the two energetic minima of $\mathrm{Mn}$ from $\Delta E=0.05 \mathrm{eV}$ in the isolated case to $\Delta E=$ $0.28 \mathrm{eV}$ for the $1 \mathrm{nn}$ Mn-vacancy case. This behavior may be qualitatively understood by a local electronic redistribution. The presence of a nearby vacancy induces a depletion of the Mn charge (see Fig. 19), which is expected to favor further the

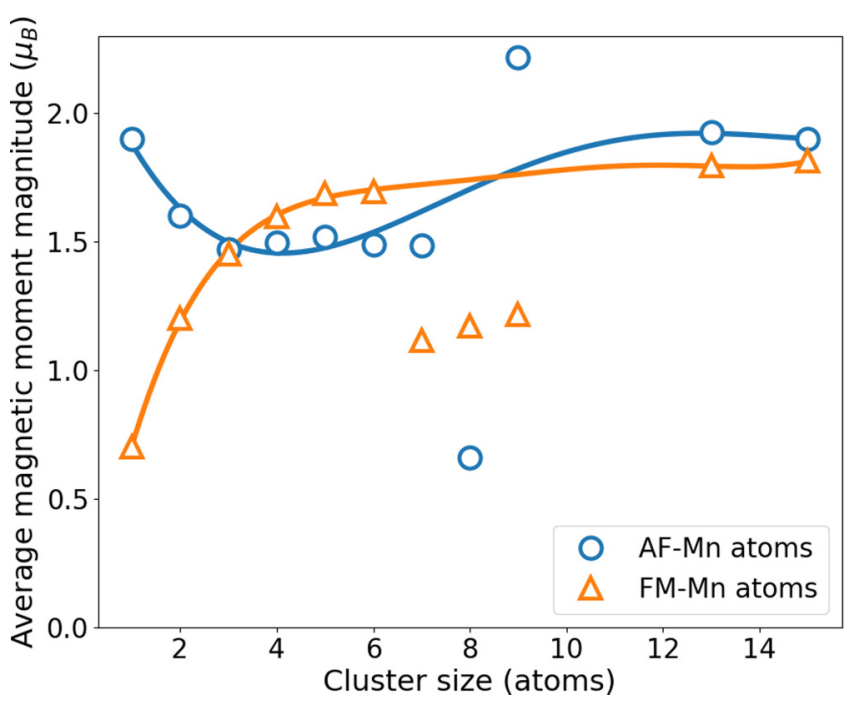

FIG. 12. Average magnetic moment magnitude of $\mathrm{Mn}$ atoms coupled AF (FM) with Fe atoms as a function of the number of Mn atoms composing the cluster. Solid lines are polynomials fitted on the data points which follow a general trend (see text), in order to evidence the behavior of the intermediate-size points which deviate from this trend. 


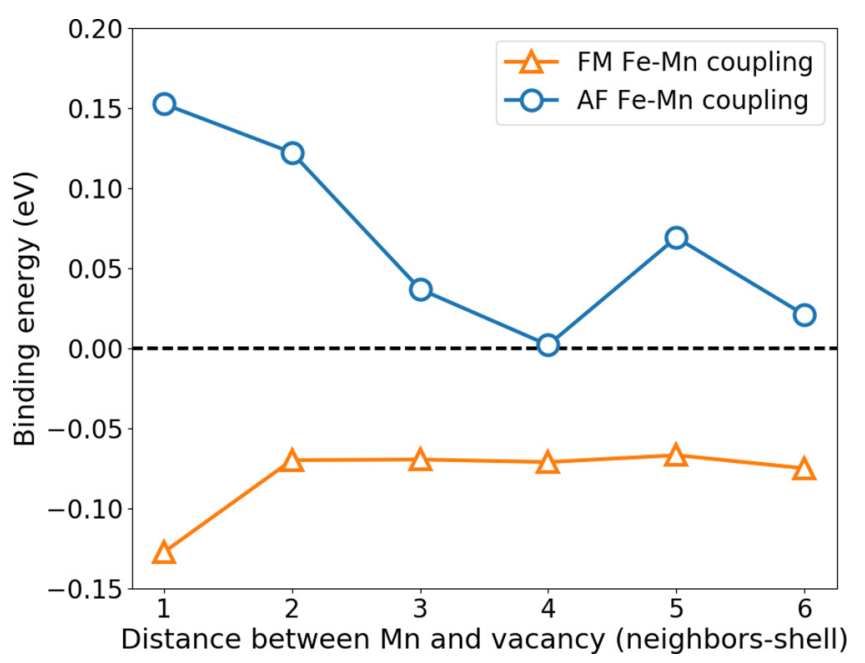

FIG. 13. Binding energy between a vacancy and an isolated Mn solute in bcc Fe as a function of their separation, taking the AF-Mn as reference. Positive values mean attraction.

AF-Mn state compared to the FM-Mn state. In addition, lattice distortion considerations can also contribute to rationalize the same behavior, since the FM-Mn has a lower effective volume than the AF-Mn (see Sec. III A). We estimated in both cases the lattice distortion energy by replacing both the vacancy and the Mn solute in the relaxed supercell by Fe atoms, without further optimizing the atomic positions. The lattice distortion energy is calculated as the difference between the resulting supercell energy and the energy of a perfect bulk bcc Fe with the same number of atoms. This energy is respectively 0.36 and $0.30 \mathrm{eV}$ for the FM-Mn and AF-Mn case. In comparison, the lattice distorsion energy for an AF-Mn and a FM-Mn, without the vacancy is practically the same, differing in only $0.005 \mathrm{eV}$.

The binding energy between the vacancy and the Mn solute was calculated with the following expression:

$$
\begin{aligned}
E^{\mathrm{bind}}(V-\mathrm{Mn})= & -E^{\mathrm{tot}}((n-2) \mathrm{Fe}+\mathrm{Mn}+V) \\
& +E^{\mathrm{tot}}((n-1) \mathrm{Fe}+1 \mathrm{Mn}) \\
& +E^{\mathrm{tot}}((n-1) \mathrm{Fe}+V)-E^{\mathrm{tot}}(n \mathrm{Fe}),
\end{aligned}
$$

with $E^{\text {tot }}(n \mathrm{Fe})$ being the total energy of a pure $n$-atoms $\mathrm{Fe}$ system, $E^{\text {tot }}((n-1) \mathrm{Fe}+V)$ the total energy of a $n$-atoms $\mathrm{Fe}$ system with one of the Fe atoms replaced by a vacancy $(V), E^{\text {tot }}((n-1) \mathrm{Fe}+1 \mathrm{Mn})$ the total energy of a $n$-atoms Fe system with one of the Fe atoms replaced by an AF-Mn atom, and $E^{\text {tot }}((n-2) \mathrm{Fe}+\mathrm{Mn}+V)$ the total energy of a $n$-atom Fe system containing both a Mn atom and a vacancy. As shown in Fig. 13, there is a strong attraction between the vacancy and the AF-Mn at a short range (from $1 \mathrm{nn}$ to $3 \mathrm{nn}$ ) which decays to zero at longer distances except for the $5 \mathrm{nn}$ case. The relatively high value for the $5 \mathrm{nn}$ case may be due to the fact that, in a bcc lattice, it is actually the second-nearest neighbor in the densest (111) direction. This feature is however not observed in the case of FM-Mn, which has a smaller effective volume than Fe (see Sec. III A).

The insertion of a vacancy has a noticeable impact on the interaction between two Mn solutes in bcc Fe. Our
TABLE V. Magnetic and energetic effects of a vacancy on a Mn dimer. The first column shows the distance between two Mn atoms and the second column shows their magnetic moments in the presence of vacancy. The binding energies between $\mathrm{Mn}$ atoms with and without vacancy are given respectively in the third and fourth columns. Finally, the total and pairwise binding energies are given respectively in the fifth and sixth columns. Pairwise binding energy is calculated as the sum of $\mathrm{Mn}-\mathrm{Mn}$ and $\mathrm{Mn}-\mathrm{V}$ binding energies for the two $\mathrm{Mn}$ atoms. Lowest energy magnetic states are taken as references for every binding energy calculation.

\begin{tabular}{lccccc}
\hline \hline $\begin{array}{l}\text { Mn-Mn } \\
\text { dist. }\end{array}$ & $\begin{array}{c}\mu_{\mathrm{Mn}} \\
\left(\mu_{B}\right)\end{array}$ & $\begin{array}{c}E_{\text {bind }} \\
(\mathrm{MnV}-\mathrm{Mn})\end{array}$ & $\begin{array}{c}E_{\text {bind }} \\
(\mathrm{Mn}-\mathrm{Mn})\end{array}$ & $E_{\text {bind }}^{\text {tot }}$ & $E_{\text {bind }}^{\text {pair }}$ \\
\hline $1 \mathrm{nn}$ & $-2.87 /$ & $0.058 \mathrm{eV}$ & $0.012 \mathrm{eV}$ & $0.181 \mathrm{eV}$ & $0.287 \mathrm{eV}$ \\
& -2.29 & & & & \\
$2 \mathrm{nn}$ & -2.63 & $0.108 \mathrm{eV}$ & $-0.032 \mathrm{eV}$ & $0.261 \mathrm{eV}$ & $0.273 \mathrm{eV}$ \\
$3 \mathrm{nn}$ & -2.74 & $0.208 \mathrm{eV}$ & $-0.044 \mathrm{eV}$ & $0.361 \mathrm{eV}$ & $0.261 \mathrm{eV}$ \\
$5 \mathrm{nn}$ & -2.67 & $0.171 \mathrm{eV}$ & $-0.060 \mathrm{eV}$ & $0.324 \mathrm{eV}$ & $0.245 \mathrm{eV}$ \\
\hline \hline
\end{tabular}

calculations show that when the Mn atoms are surrounding a vacancy, they tend to couple each AF with $\mathrm{Fe}$ atoms of the matrix, regardless the distance between those solutes $(2 \mathrm{nn}$, $3 \mathrm{nn}$, or $5 \mathrm{nn}$ ). As expected, the magnetic moment magnitude of these $\mathrm{Mn}$ atoms is significantly increased compared to the corresponding configuration without the vacancy, due to the magnetovolume effect (see Table V). One can also notice that among the atomic configurations considered, two $\mathrm{Mn}$ atoms located at the first-neighbor shell of a vacancy prefer to arrange as $3 \mathrm{nn}$ to each other. The second lowest energy configuration being as $5 \mathrm{nn}$ to each other, followed by the $2 \mathrm{nn}$ case which is the worst arrangement in terms of system energy. Although it is shown in Fig. 8 that $2 \mathrm{nn}$ $\mathrm{Mn}$ are less repulsive than $3 \mathrm{nn} \mathrm{Mn}$ in their respective lowest energy magnetic configuration, the presence of the vacancy stabilizes the AF-Mn state and thus $2 \mathrm{nn}$ Mn atoms become more repulsive because they are constrained in a higher energy magnetic configuration.

The binding energy between two Mn atoms was compared with and without the presence of a vacancy as $1 \mathrm{nn}$ of both $\mathrm{Mn}$ atoms. Except in the case of $1 \mathrm{nn} \mathrm{Mn}$ atoms, where the vacancy is inserted as respectively $1 \mathrm{nn}$ and $2 \mathrm{nn}$ of the $\mathrm{Mn}$ solutes. In all the studied cases, the vacancy either promote or enhance attraction between the Mn atoms (Table V). Also, the comparison between the total binding energy and the pairwise binding energy reveals a synergistic effect in the cases of $3 \mathrm{nn}$ and 5nn Mn dimers. Pairwise binding energy is calculated as the sum of Mn-Mn and Mn-V binding energies for the two Mn atoms. The opposite trend is observed in the cases of $1 \mathrm{nn}$ and $2 \mathrm{nn} \mathrm{Mn}$ atoms, which is consistent with a magnetic frustration induced by the vacancy stabilizing both Mn atoms as AF-Mn, while $\mathrm{Mn}-\mathrm{Mn}$ interactions are favoring a different magnetic state (AF-Mn + FM-Mn.)

In order to study the effect of a vacancy on Mn clusters magnetic properties, further calculations were performed with a vacancy in bcc Fe while replacing, atom by atom, its first nearest neighbors by $\mathrm{Mn}$ atoms, as shown in Fig. 15. For each number of $\mathrm{Mn}$ atoms, various atomic configurations were studied and for each of them, several magnetic states were considered. More open cluster configurations were also 


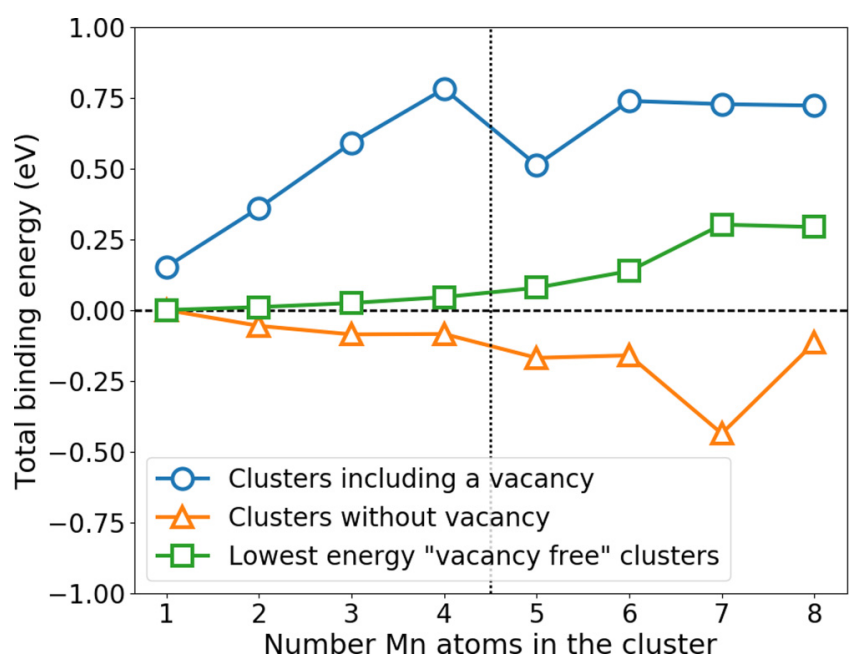

FIG. 14. Comparison of total binding energies of: (1) clusters composed of one vacancy and $n$-Mn atoms. The corresponding structures are shown in Fig. 15. (2) Clusters with the same Mn arrangement and the vacant site replaced by a Fe atom. (3) The lowest-energy $n$-Mn clusters found. The isolated AF-Mn is taken as reference.

tested, that is, locating some $\mathrm{Mn}$ atoms on the $2 \mathrm{nn}$ shell of the vacancy instead of being $1 \mathrm{nn}$ of it. The most compact configurations are always found to be the most energetically favorable.

The first observation is that the vacancy tends to stabilize the AF coupling of Mn atoms with Fe atoms of the matrix. Indeed, every atomic configuration tends to exhibit a full $\mathrm{AF}$ Fe-Mn coupling while corresponding vacancy-free clusters show more complex spin configurations (see Sec. III B).

Two complementary effects may explain this observation. As previously shown in the case of a single $\mathrm{Mn}$ atom, the vacancy tends to stabilize the AF-Mn state. It also acts as a magnetic screener reducing the impact of the Mn-Mn interactions described in Sec. III C. Such a behavior is also observed in the case of $\mathrm{Cr}$ atoms near a vacancy in bcc $\mathrm{Fe}$ [64].

The total binding energy between the vacancy and every $\mathrm{Mn}$ atom of the cluster is calculated for the lowest energy atomic and magnetic configuration found for each number of Mn atoms, using the following formula:

$$
\begin{aligned}
E^{\mathrm{bind}}(V-p \mathrm{Mn})= & -E^{\mathrm{tot}}((n-p-1) \mathrm{Fe}+p \mathrm{Mn}+V) \\
& +p E^{\mathrm{tot}}((n-1) \mathrm{Fe}+\mathrm{Mn}) \\
& +E^{\mathrm{tot}}((n-1) \mathrm{Fe}+V)-p E^{\mathrm{tot}}(n \mathrm{Fe}),
\end{aligned}
$$

with $E^{\text {tot }}(n \mathrm{Fe})$ being the total energy of a pure $n$-Fe bcc bulk, $E^{\text {tot }}((n-1) \mathrm{Fe}+V)$ the total energy of the $n$-Fe system with one of the $\mathrm{Fe}$ atoms replaced by a vacancy, $E^{\text {tot }}((n-1) \mathrm{Fe}+\mathrm{Mn})$ the total energy of a $n$-Fe system with one of the $\mathrm{Fe}$ atoms replaced by a $\mathrm{Mn}$ atom, and $E^{\text {tot }}((n-p-1) \mathrm{Fe}+p \mathrm{Mn}+V)$ the total energy of a system containing $p \mathrm{Mn}$ atoms and a vacancy.

The results shown in Fig. 14 reveal that the total binding energy is positive and increases with the number of Mn atoms up to $4 \mathrm{Mn}$. Then it reaches a plateau and the binding energy

\section{- $\mathrm{Mn}$ \\ - $\mathrm{Fe}$ \\ [1] Vac}

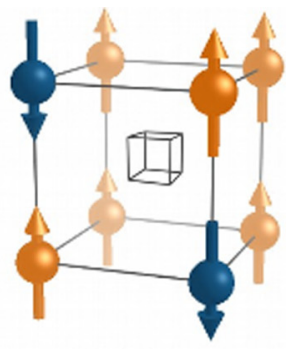

$2 \mathrm{Mn}$
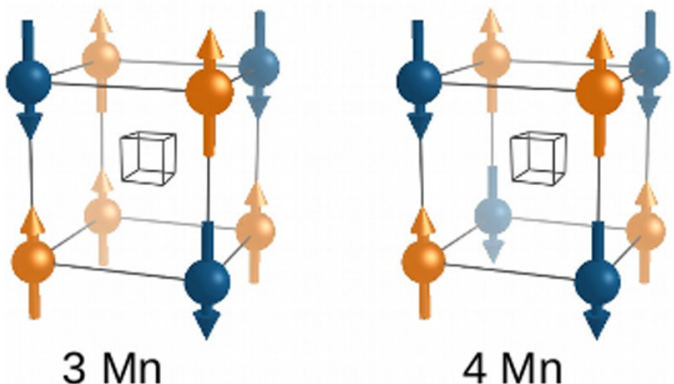

$4 \mathrm{Mn}$

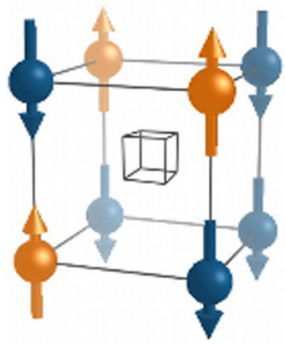

$5 \mathrm{Mn}$

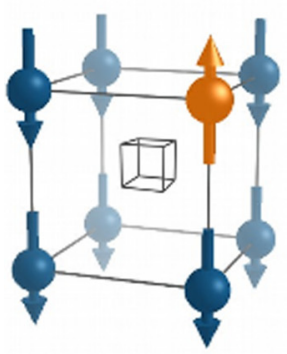

$7 \mathrm{Mn}$

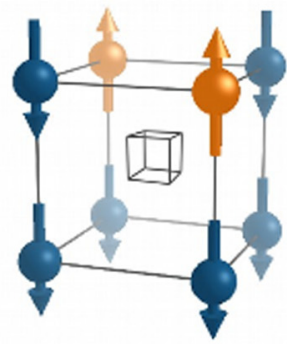

$6 \mathrm{Mn}$

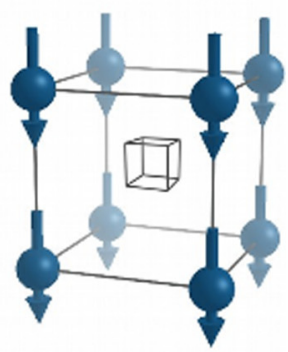

$8 \mathrm{Mn}$
FIG. 15. Schematic representation of the lowest energy atomic and magnetic configuration of Mn clusters in bcc Fe composed of a vacancy surrounded by $n \mathrm{Mn}$ atoms.

remains constant between 6 and $8 \mathrm{Mn}$ cases. We note a rather small value for the 5-Mn case. It can be explained as follows: Up to $4 \mathrm{Mn}$ atoms on the $1 \mathrm{nn}$ shell of the vacancy, the lowestenergy atomic configuration is such as the Mn atoms are $3 \mathrm{nn}$ to each other (the most favorable state for two Mn atoms, see Fig. 15). From the fifth $\mathrm{Mn}$ atom introduced to the $1 \mathrm{nn}$ shell, such an arrangement is no longer possible and some Mn atoms have to be a $2 \mathrm{nn}$, which has a higher energy as seen in the case of a Mn dimer around a vacancy.

Another relevant feature is that the binding energy is much higher than in the corresponding vacancy-free Mn clusters, 


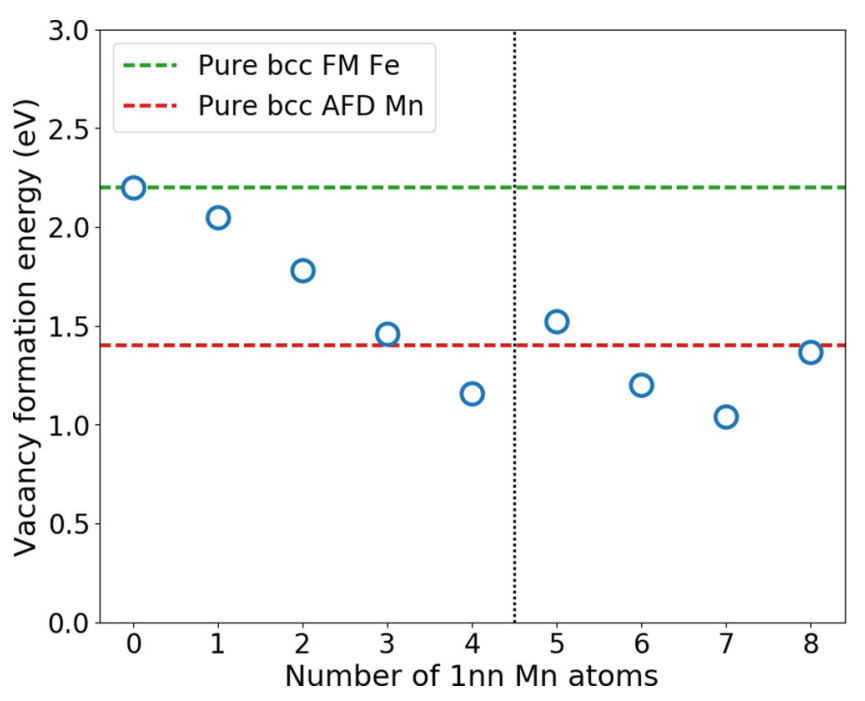

FIG. 16. Vacancy formation energy as a function of the number of $\mathrm{Mn}$ atoms on its first-neighbor shell.

which indicates an enhancement of Mn clustering due to the presence of vacancies, as also noticed in $\mathrm{Fe}-\mathrm{Cr}$ alloys [64]. This behavior may be relevant in Fe-Mn systems under some extreme conditions with creation of large amounts of vacancies.

The vacancy formation energy was also evaluated as a function of the number of $\mathrm{Mn}$ atoms at the $1 \mathrm{nn}$ shell of the vacancy. Figure 16 shows that the vacancy formation energy decreases almost linearly with the number of $\mathrm{Mn}$ atoms up to $4 \mathrm{Mn}$ atoms, then it oscillates around the value found in the pure bcc AFD-Mn bulk. This decreasing trend suggests that the vacancy formation is more favorable in a Mn-rich than in a Fe-rich local environment in Fe-Mn alloys. In addition, similarly to the bcc $\mathrm{Fe}-\mathrm{Cu}$ alloys [65], the mobility of $\mathrm{Mn}$ clusters may be significantly enhanced due to the preference of vacancies to be located around a Mn cluster than in the Fe matrix, dictated by the difference of respective vacancy formation energies.

The present study is limited to the case of $\mathrm{Mn}$ atoms around a single vacancy. The effects of multiple vacancies may also be relevant, especially for the understanding of the Fe-Mn alloys under extreme conditions such as plastic deformation or irradiation. It is however beyond the scope of this paper.

\section{E. Interstitial impurities and $\mathrm{Mn}$ in bcc $\mathrm{Fe}$}

Carbon, nitrogen, and oxygen atoms, neighbors in the periodic table of elements, are highly common interstitial impurities in iron-based alloys. It is thus important to understand the interaction between these elements and Mn solutes in bcc $\mathrm{Fe}$, in order to clarify the impact of these interstitial atoms on magnetic properties of Mn solutes.

In this study we only consider the effect of a single interstitial atom, located at the lowest energy octahedral site [66], near a Mn atom. We first evaluated the interaction energy between a single Mn solute and a foreign interstitial atom $(\mathrm{FIA}=\mathrm{C}, \mathrm{N}$, or $\mathrm{O})$, at a $1 \mathrm{nn}$ distance. The binding energy between the 1nn FIA and the Mn solute in the lowest-energy magnetic configuration is attractive in the three cases $(0.08 \mathrm{eV}$ for $\mathrm{C}, 0.21 \mathrm{eV}$ for $\mathrm{N}$, and 0.24 for $\mathrm{O}$ ). Interestingly, in the
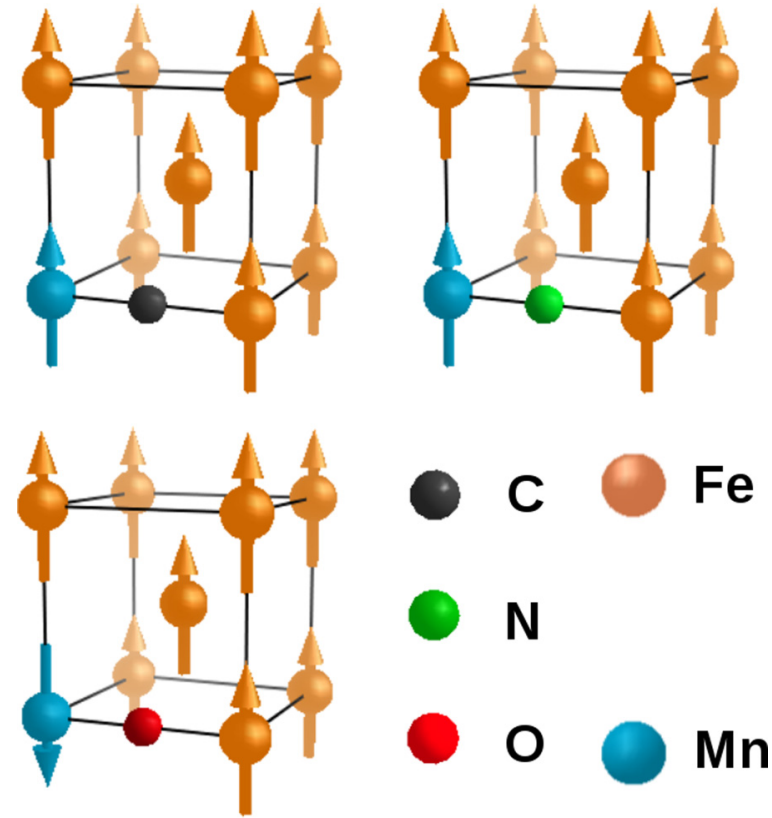

FIG. 17. Schematic representation of the lowest energy magnetic configuration of a $\mathrm{Mn}$ atom in bcc $\mathrm{Fe}$, with a $\mathrm{X}$ interstitial at its $1 \mathrm{nn}$ octahedral site $(\mathrm{X}=\mathrm{C}, \mathrm{N}, \mathrm{O})$.

case of $\mathrm{C}$ and $\mathrm{N}$ atoms, the $\mathrm{Mn}$ magnetic moment tends to be parallel to the $\mathrm{Fe}$ moments (opposite to the case of isolated $\mathrm{Mn}$ solutes), whereas in the case of an $\mathrm{O}$ atom, $\mathrm{Mn}$ moment tends to be antiparallel to the Fe moments, as shown in Fig. 17. The results for the case of $\mathrm{C}$ are in good agreement with a previous DFT study, on both Mn-C binding energy and Mn magnetic moment $[63,67]$.

Projected densities of states shown in Fig. 18 reveal a strong hybridization between the $p$ shell of $\mathrm{C}$ atom and the $d$ shell of Mn. As expected, with increasing $p$-band filling of the FIAs, such hybridization is weaker in the case of the $\mathrm{N}$ interstitial and negligible in the case of the $\mathrm{O}$ interstitial. The right panel of the same figure shows differential charge density maps obtained by subtracting the charge density of the isolated $\mathrm{C}, \mathrm{N}$, or $\mathrm{O}$ atom and the Fe-Mn system without interstitial defect, to the same Fe-Mn fully relaxed system containing the FIA. Note that the atomic positions are kept identical for the subtractions. The charge maps show that, as expected, $\mathrm{C}$ exhibits a covalent bond with the $\mathrm{Mn}$ atom, increasing the local charge density around the $\mathrm{Mn}$ atom, whereas the charge density around the $\mathrm{O}$ impurity remains very localized, which explains that $\mathrm{O}$ has no noticeable effect on the magnetic coupling of the $\mathrm{Mn}$ atom. The $\mathrm{N}$ interstitial shows an intermediate behavior. As stated in the Introduction and in Sec. III A, the magnetic state of Mn is especially flexible in bcc iron, reflected for instance by the presence of two minima (AF-Mn and FM-Mn) for the single solute. In order to adapt to different local environments, such as in the Mn dimer, the solute may easily change its preferred magnetic state. In the case of a Mn solute next to a $\mathrm{C}$ or a $\mathrm{N}$ interstitial, the interstitial atom induces a $p$ - $d$ hybridization which strongly increases the local charge density around the Mn atom (Fig. 18), and leads to a significant change in the PDOS of the Mn atom. Both up- and down-spin states 

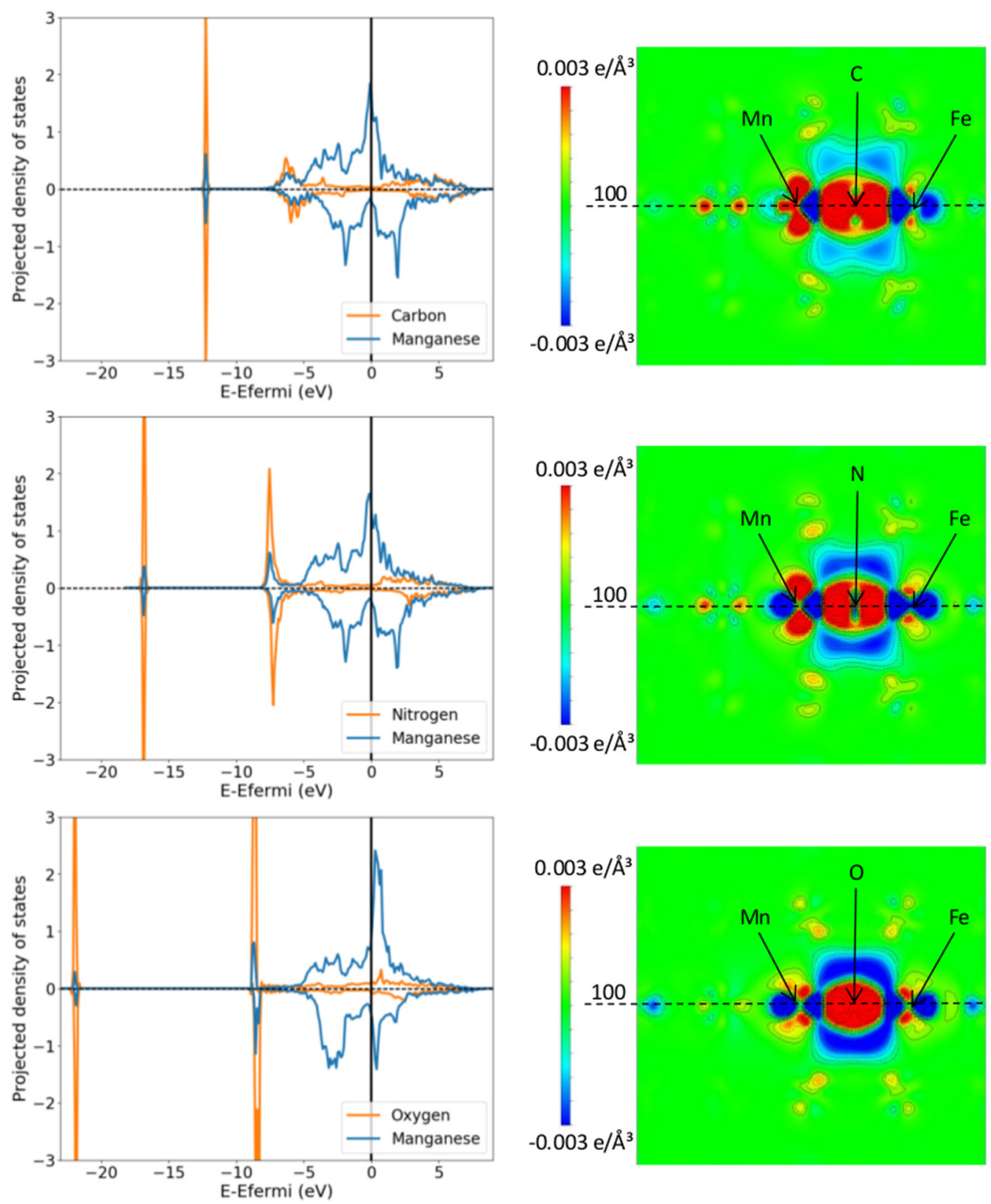

FIG. 18. Projected density of states on Mn atom and X impurity, X being C, N, or O (left), and the corresponding differential charge density maps (right). Further details are given in the text.

approach those of the isolated FM-Mn PDOS (Fig. 2). For example, the up-spin PDOS of an isolated AF-Mn atom (without the interstitial) shows a sharp peak just above the Fermi level, which is shifted down toward the Fermi level when the local charge increases (with the interstitial). Then, in order to reduce the density at the Fermi level, the peak becomes splited. As a consequence, the Mn magnetic state switches to the FM-Mn energy minimum, which has a higher local charge density.

The impact of the FIAs on the magnetic state of a Mn solute is rather short ranged. If the FIA is inserted in the $2 \mathrm{nn}$ octahedral site of a Mn atom, the magnetic moment of the $\mathrm{Mn}$ atom remains antiparallel to the lattice $\mathrm{Fe}$ moments, the impurity being $\mathrm{C}, \mathrm{N}$, or $\mathrm{O}$. In this case, the Mn-FIA binding energy is $-0.20 \mathrm{eV}$ in the case of $\mathrm{C},-0.11 \mathrm{eV}$ for $\mathrm{N}$, and
0.04 for O. Clearly the hierarchy of these energies follows the same trend as for the $1 \mathrm{nn}$ interactions. Here too, the results in the case of $\mathrm{C}$ are consistent with the literature [67].

Due to the expected lattice distortion induced by the interstitial atoms, all the presented results are also verified by complementary fully relaxed calculations. We checked that the relevant results present only minor changes compared to the constant-volume results: variations are in any case lower than $0.01 \mu_{\mathrm{B}}$ for the magnetic moment and lower than $10 \mathrm{meV}$ for the binding energy.

Based on the results presented in previous subsections, a correlation can be identified between the magnetic moment of a single Mn solute in bcc Fe and its local charge density. Indeed, the presence of a vacancy, inducing a local charge decrease, tends to favor the AF Fe-Mn magnetic coupling 


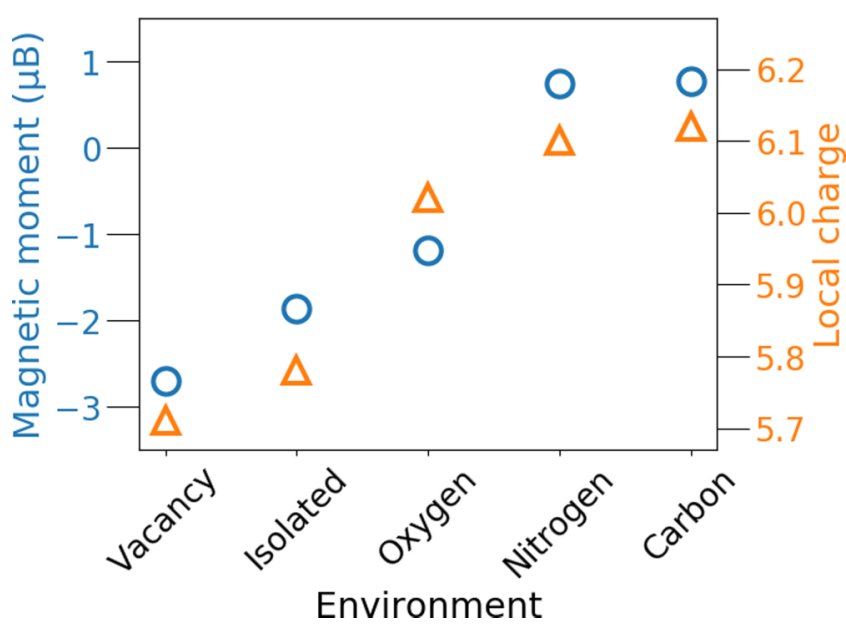

FIG. 19. Correlation between local magnetic moment (triangles) on a $\mathrm{Mn}$ atom and its local charge density (circles) for various chemical environments: isolated in $\mathrm{Fe}, 1 \mathrm{nn}$ of a vacancy, and $1 \mathrm{nn}$ of a $\mathrm{X}$ interstitial $(\mathrm{X}=\mathrm{C}, \mathrm{N}, \mathrm{O})$.

while the presence of an interstitial impurity (particularly the C) may favor the FM Fe-Mn coupling. This trend is illustrated in Fig. 19 where the magnetic moment and the local charge of the $\mathrm{Mn}$ solute are plotted for various local environments. Both local charge and magnetic moment are obtained by integrating the Mn PDOS, although the absolute values may depend on the integration criterion chosen, the relative values show a clear trend.

In the case of Mn clusters, the Mn-Mn interactions being dominant over the Fe-Mn interactions, this trend is not general. However, for the Mn dimer and the smallest sizes of clusters (up to $4 \mathrm{Mn}$ atoms), the local charge of an AF-Mn is still lower than the charge of a FM-Mn, as shown in Fig. 20. Indeed, Mn atoms in these small clusters have a majority of Fe neighbors.

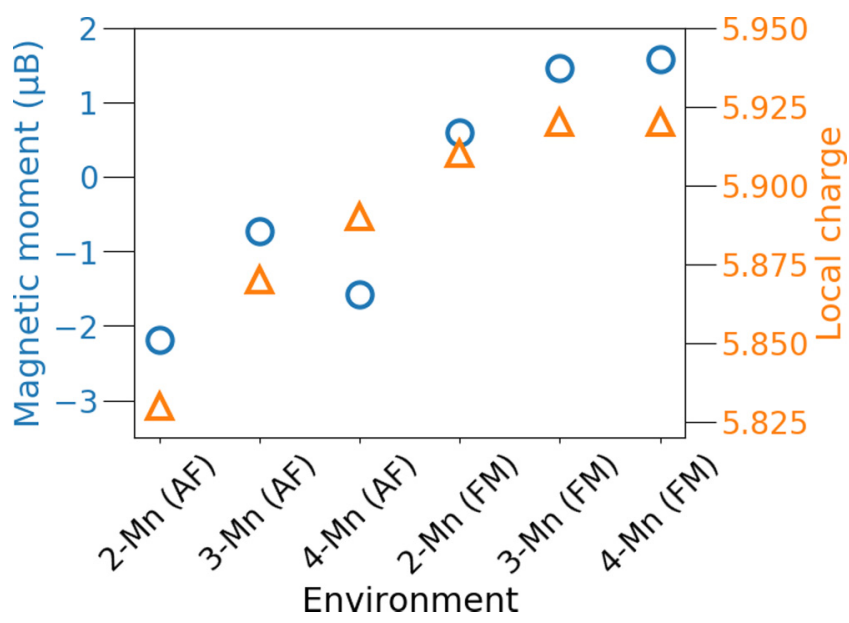

FIG. 20. Correlation between local magnetic moment (triangles) on $\mathrm{Mn}$ and the corresponding local charge density (circles). Each moment or charge value results from an average over all the $\mathrm{Mn}$ atoms showing the same spin orientation in a $n$-Mn cluster. For example "4-Mn (FM)" in the $x$ axis means $\mathrm{Mn}$ atoms in a 4-Mn cluster, showing a FM interaction with $\mathrm{Fe}$ atoms.

\section{CONCLUSIONS}

Density functional theory calculations are applied to investigate magnetic and energetic properties of $\mathrm{Mn}$ in bcc Fe-Mn alloys, as functions of the alloy concentration and local chemical environment. Manganese, with a half-filled $3 d$ band, exhibits an atypical and complex magnetic behavior.

For the simplest case of an isolated $\mathrm{Mn}$ in bcc Fe, we confirm the presence of two collinear energy minima, with the Mn magnetic moment either parallel or antiparallel to the Fe moments, named respectively the FM-Mn and the AF-Mn state. The latter being the ground state. Interestingly, the preference of the Fe-Mn magnetic interaction is highly sensitive to minor changes of the Mn local environment, due to the presence of a high Mn majority-spin electronic density around the Fermi level.

A direct correlation is identified between the local electronic charge and the local magnetic moment on a Mn solute, being isolated or forming a small $n$-Mn cluster ( $n=2$ to 4). For instance, the presence of a vacancy near the $\mathrm{Mn}$ atom, inducing a local charge depletion, tends to favor the antiferromagnetic Fe-Mn interaction. Also, common interstitial impurities $(\mathrm{C}, \mathrm{N}$, and $\mathrm{O}$ ) present an attraction with a $\mathrm{Mn}$ atom if located at its $1 \mathrm{nn}$ site. $\mathrm{C}$ and $\mathrm{N}$ show a strong electronic hybridization with $\mathrm{Mn}$ and stabilize the FM-Mn state. At variance, an oxygen atom, with a rather ionic $\mathrm{Mn}-\mathrm{O}$ interaction and therefore a very localized charge distribution, does not modify the magnetic ground state of an isolated Mn.

Properties of small $n$-Mn clusters ( $n=2$ to 15 ) in dilute $\mathrm{Fe}-\mathrm{Mn}$ systems are analyzed. Clustering is found to be energetically favorable, consistently with the unmixing tendency found for the bcc Fe-Mn alloys. Various collinear and noncollinear magnetic minima are found for the Mn dimer, which are rather close in energy. It is relevant to point out that our results suggest a dominance of $\mathrm{Mn}-\mathrm{Mn}$ magnetic interactions over the Fe-Mn interactions, both exhibiting an antiferromagnetic tendency, especially for the $1 \mathrm{nn}$ and $2 \mathrm{nn}$ distances. This behavior is opposite to the $\mathrm{Fe}-\mathrm{Cr}$ alloy case, where $\mathrm{Fe}-\mathrm{Cr}$ $\mathrm{AF}$ interactions prevail over the $\mathrm{Cr}-\mathrm{Cr}$ antiferromagnetism. A vacancy is found to further stabilize the Mn clusters, by increasing the clusters binding energies. Reciprocally, the formation energy of a vacancy becomes smaller in a $\mathrm{Mn}$ rich than in a Fe-rich local environment. This behavior may promote a rather fast diffusion of Mn nanoclusters, similar to the case of bcc $\mathrm{Fe}-\mathrm{Cu}$ alloys.

Locally random (SQS) Fe-Mn solid solutions are systematically investigated for a large range of $\mathrm{Mn}$ concentrations. Consistently with experimental findings, the average magnetic moment of the alloys decreases linearly with increasing $\mathrm{Mn}$ content. In addition, the average Mn moment tends to be antiparallel (parallel) to lattice $\mathrm{Fe}$ moments for $\mathrm{Mn}$ concentrations smaller (larger) than approximately 6 at. \% Mn. The same trend is observed experimentally, but the deduced transition concentration is around 2 at. \%. Various possible reasons for this discrepancy are discussed. In particular, the calculated transition concentration is lowered if considering Mn clustering. Indeed Mn clusters may be present in the experimental Fe-Mn samples, depending on the applied heat treatment. 


\section{ACKNOWLEDGMENTS}

This study is partially supported by the French-German ANR-DFG MAGIKID project. This work was performed using DARI-GENCI resources under the A0030906020 project, and the CINECA-MARCONI supercomputer within the SISTEEL project. We thank Dr. T. Hickel and Dr. O. Waseda for fruitful discussions.
[1] V. I. Anisimov, V. P. Antropov, A. I. Liechtenstein, V. A. Gubanov, and A. V. Postnikov, Phys. Rev. B 37, 5598 (1988).

[2] S. Blügel, H. Akai, R. Zeller, and P. H. Dederichs, Phys. Rev. B 35, 3271 (1987).

[3] R. Zeller, J. Phys. F: Met. Phys. 17, 2123 (1987).

[4] P. Olsson, I. A. Abrikosov, L. Vitos, and J. Wallenius, J. Nucl. Mater. 321, 84 (2003).

[5] P. Olsson, I. A. Abrikosov, and J. Wallenius, Phys. Rev. B 73, 104416 (2006)

[6] P. Olsson, C. Domain, and J. Wallenius, Phys. Rev. B 75, 014110 (2007).

[7] T. P. C. Klaver, R. Drautz, and M. W. Finnis, Phys. Rev. B 74, 094435 (2006)

[8] R. Soulairol, C.-C. Fu, and C. Barreteau, Phys. Rev. B 84, 155402 (2011).

[9] M. Yu. Lavrentiev, R. Soulairol, C.-C. Fu, D. Nguyen-Manh, and S. L. Dudarev, Phys. Rev. B 84, 144203 (2011).

[10] C.-C. Fu, M. Y. Lavrentiev, R. Soulairol, S. L. Dudarev, and D. Nguyen-Manh, Phys. Rev. B 91, 094430 (2015).

[11] R. Soulairol, C. Barreteau, and C.-C. Fu, Phys. Rev. B 94, 024427 (2016).

[12] A. V. Ruban and V. I. Razumovskiy, Phys. Rev. B 86, 174111 (2012).

[13] O. Senninger, E. Martínez, F. Soisson, M. Nastar, and Y. Bréchet, Acta Mater. 73, 97 (2014).

[14] D. Hobbs, J. Hafner, and D. Spišák, Phys. Rev. B 68, 014407 (2003).

[15] J. Hafner and D. Hobbs, Phys. Rev. B 68, 014408 (2003).

[16] D. Music, T. Takahashi, L. Vitos, C. Asker, I. A. Abrikosov, and J. M. Schneider, Appl. Phys. Lett. 91, 191904 (2007).

[17] O. Schneeweiss, M. Friák, M. Dudová, D. Holec, M. Šob, D. Kriegner, V. Holý, P. Beran, E. P. George, J. Neugebauer et al., Phys. Rev. B 96, 014437 (2017).

[18] V. T. Witusiewicz, F. Sommer, and E. J. Mittemeijer, J. Phase Equilib. Diffus. 25, 346 (2004).

[19] A. S. Nikonenko, Fiz. Metal. Metalloved 16, 776 (1963).

[20] S. Lintzen, J. von Appen, B. Hallstedt, and R. Dronskowski, J. Alloys Compd. 577, 370 (2013).

[21] A. A. Mirzoev, M. M. Yalalov, and D. A. Mirzaev, Phys. Met. Metallogr. 101, 341 (2006).

[22] N. I. Kulikov and C. Demangeat, Phys. Rev. B 55, 3533 (1997).

[23] P. Olsson, T. P. C. Klaver, and C. Domain, Phys. Rev. B 81, 054102 (2010).

[24] A. Bakaev, D. Terentyev, G. Bonny, T. P. C. Klaver, P. Olsson, and D. Van Neck, J. Nucl. Mater. 444, 237 (2014).

[25] E. Vincent, C. S. Becquart, and C. Domain, J. Nucl. Mater. 351, 88 (2006).

[26] L. Messina, M. Nastar, T. Garnier, C. Domain, and P. Olsson, Phys. Rev. B 90, 104203 (2014).

[27] E. Vincent, C. S. Becquart, and C. Domain, Nucl. Instrum. Methods B 228, 137 (2005).
[28] A. Moslang, E. Albert, E. Recknagel, A. Weidinger, and P. Moser, Hyperfine Interact. 15, 409 (1983).

[29] M. Doyama, Trans. Jpn. Inst. Met. 25, 808 (1986).

[30] Y. You and M. F. Yan, Physica B 417, 57 (2013).

[31] V. A. Shabashov, K. A. Kozlov, V. V. Sagaradze, A. L. Nikolaev, K. A. Lyashkov, V. A. Semyonkin, and V. I. Voronin, Philos. Mag. 20, 560 (2017).

[32] G. Parette, J. Appl. Phys. 69, 6135 (1991).

[33] S. G. Kang, H. Onodera, H. Yamamoto, and H. Watanabe, J. Phys. Soc. Jpn. 36, 975 (1974).

[34] G. K. Wertheim, V. Jaccarino, J. H. Wernick, and D. N. E. Buchanan, Phys. Rev. Lett. 12, 24 (1964).

[35] M. B. Stearns and S. S. Wilson, Phys. Rev. Lett. 13, 313 (1964).

[36] V. Jaccarino, L. R. Walker, and G. K. Wertheim, Phys. Rev. Lett. 14, 89 (1965).

[37] D. A. Shirley, S. S. Rosenblum, and E. Mathias, Phys. Rev. 170, 363 (1968)

[38] M. Rubinstein, G. H. Strauss, and J. Dweck, Phys. Rev. Lett. 17, 41 (1966).

[39] M. F. Collins and G. G. Low, Proc. Phys. Soc. 86, 535 (1965).

[40] H. Yamauchi, H. Watanabe, Y. Suzuki, and H. Saito, J. Phys. Soc. Jpn. 36, 971 (1974).

[41] C. Paduani, E. Galvao da Silva, G. A. Perez-Alcazar, and M. McElfresh, J. Appl. Phys. 70, 7524 (1991).

[42] H. Bhatkar, R. J. Snow, E. Arenholz, and Y. U. Idzerda, J. Magn. Magn. Mater. 423, 46 (2017).

[43] F. Kajzar and G. Parette, Phys. Rev. B 22, 5471 (1980).

[44] Y. Nakai and N. Kunitomi, J. Phys. Soc. Jpn. 39, 1257 (1975).

[45] H. R. Child and J. W. Cable, Phys. Rev. B 13, 227 (1976).

[46] P. Radhakrishna and F. Livet, Solid State Commun. 25, 597 (1978)

[47] S. Gosh, B. Sanya, C. B. Chaudhuri, and A. Mookerjee, Eur. Phys. J. B 23, 455 (2001).

[48] D. J. M. King, S. C. Middleburgh, P. A. Burr, T. M. Whiting, P. C. Fossati, and M. R. Wenman, Phys. Rev. B 98, 024418 (2018).

[49] P. E. Blöchl, Phys. Rev. B 50, 17953 (1994).

[50] G. Kresse and D. Joubert, Phys. Rev. B 59, 1758 (1999).

[51] G. Kresse and J. Hafner, Phys. Rev. B 47, 558 (1993).

[52] G. Kresse and J. Furthmüller, Comp. Mat. Sci. 6, 15 (1996).

[53] G. Kresse and J. Furthmüller, Phys. Rev. B 54, 11169 (1996).

[54] J. P. Perdew, K. Burke, and M. Ernzerhof, Phys. Rev. Lett. 77, 3865 (1996).

[55] A. Zunger, S.-H. Wei, L. G. Ferreira, and J. E. Bernard, Phys Rev. Lett. 65, 353 (1990).

[56] H. J. Monkhorst and J. D. Pack, Phys. Rev. B 13, 5188 (1976).

[57] M. Methfessel and A. T. Paxton, Phys. Rev. B 40, 3616 (1989).

[58] H. C. Herper, E. Hoffmann, and P. Entel, Phys. Rev. B 60, 3839 (1999).

[59] D. E. Jiang and E. A. Carter, Phys. Rev. B 67, 214103 (2003).

[60] J. Neugebauer and T. Hickel, WIREs Comput. Mol. Sci. 3, 438 (2013). 
[61] E. G. Moroni, G. Kresse, J. Hafner, and J. Furthmüller, Phys. Rev. B 56, 15629 (1997).

[62] M. Hillert and M. Waldenstrom, Scand. J. Metall. 6, 211 (1977).

[63] N. I. Medvedeva, D. C. Van Aken, and J. E. Medvedeva, J. Phys.: Condens. Matter 23, 326003 (2011).

[64] E. Martinez and C.-C. Fu, Phys. Rev. B 84, 014203 (2011).
[65] F. Soisson and C.-C. Fu, Phys. Rev. B 76, 214102 (2007).

[66] C. Barouh, T. Schuler, C. C. Fu, and M. Nastar, Phys. Rev. B 90, 054112 (2014).

[67] P. Liu, W. Xing, X. Cheng, D. Li, Y. Li, and X.-Q. Chen, Phys. Rev. B 90, 024103 (2014). 